\title{
Light on the horizon: Current research and future perspectives in flow photochemistry Fabrizio Politano, and Gabriela Oksdath-Mansilla
}

Org. Process Res. Dev., Just Accepted Manuscript • DOI: 10.1021/acs.oprd.8b00213 • Publication Date (Web): 02 Aug 2018

Downloaded from http://pubs.acs.org on August 2, 2018

\section{Just Accepted}

"Just Accepted" manuscripts have been peer-reviewed and accepted for publication. They are posted online prior to technical editing, formatting for publication and author proofing. The American Chemical Society provides "Just Accepted" as a service to the research community to expedite the dissemination of scientific material as soon as possible after acceptance. "Just Accepted" manuscripts appear in full in PDF format accompanied by an HTML abstract. "Just Accepted" manuscripts have been fully peer reviewed, but should not be considered the official version of record. They are citable by the Digital Object Identifier (DOI®). "Just Accepted" is an optional service offered to authors. Therefore, the "Just Accepted" Web site may not include all articles that will be published in the journal. After a manuscript is technically edited and formatted, it will be removed from the "Just Accepted" Web site and published as an ASAP article. Note that technical editing may introduce minor changes to the manuscript text and/or graphics which could affect content, and all legal disclaimers and ethical guidelines that apply to the journal pertain. ACS cannot be held responsible for errors or consequences arising from the use of information contained in these "Just Accepted" manuscripts. 


\title{
Light on the horizon: Current research and
}

\section{future perspectives in flow photochemistry}

\author{
Fabrizio Politano ${ }^{[a]}$ and Gabriela Oksdath-Mansilla $*^{[a]}$
}

[a] INFIQC-CONICET-UNC, Dpto. de Química Orgánica, Facultad de Ciencias Químicas, Universidad Nacional de Córdoba, Ciudad Universitaria, X5000HUA Córdoba, Argentina, *goksdath@fcq.unc.edu.ar 


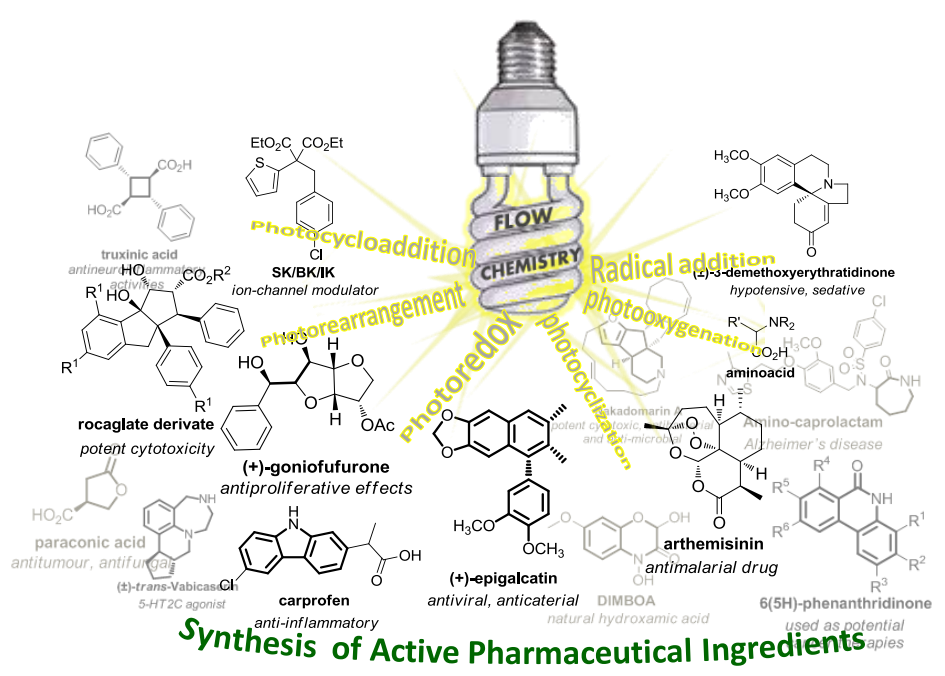

Graphical Abstract 


\section{Abstract}

The research area of synthetic organic photochemistry is a powerful tool for creating both natural products and molecules with high structural complexity, in a simple way and under mild conditions. However, because of the challenges in scaling-up, it has been difficult to apply a photochemical reaction in an industrial process. Flow chemistry provides an opportunity for better control over the conditions of the reaction and, additionally, improved reaction selectivity and enhanced reproducibility. Taking into account that significant interest has focused on the use of flow photochemistry as a method for the synthesis of heterocycles and its applications in target-oriented synthesis over the last few years, the aim of this review is to highlight the recent efforts to apply flow photochemistry methodology to diverse reactions as a greener and more scalable process for the pharmaceutical and fine chemical industries. Additionally, the review highlights future perspectives in the development of scale-up strategies, combining photochemical reactions in the continuous flow multistep synthesis of organic molecules, being of interest for scientists and engineers alike.

Keywords: flow chemistry, green processes, photochemical synthesis, APIs

\section{Introduction}

Nowadays, scientist and engineers are increasingly thinking in a sustainable way when they design their research programs. It is clear that green chemistry and engineering principles go beyond concerns over hazards from chemical toxicity and include considerations of energy conservation, waste reduction, and lifecycle such as the use of more sustainable or renewable feedstocks, and are accordingly being designed for the entire life cycle of the product. In this context, the fundamentals of photochemistry 
suggest the implementation of this process as sustainable methodology considering the photon as a green reagent which is absorbed without leaving residue. Light-induced activation offers a way for the environmentally mild obtaining of highly reactive intermediates, allowing access to molecules with valuable structural complexity which otherwise would be difficult to access. Likewise, for activation no additional reagent is required compared with thermal reaction. Therefore, photochemical transformations have been adopted in the rational and efficient design of many synthetic methodologies. $^{1}$

In spite of the numerous advantages mentioned above, different aspects should be considered when carrying out a photochemical reaction. Photophysical aspects of the reagents such as quantum yield $\left(\Phi_{\lambda}\right)$, have to be taken into account in order to increase reaction efficiency. In addition, the appropriate equipment (lamp, power supply, reaction flask with accessories, e.g. for gas inlet) are required for a successful photoreaction. In fact, there are a number of aspects in the process related to the utilization of light itself, some of them regarded as "non-green", which often reduces interest in using it. Several photoreactions involve the use of high-energy and intense light sources which are hazardous, and strict risk assessment and operation protocols are required. For powerful lamps, a forced cooling circulation system is required, and this results in a more expensive and sophisticate reactor. Inert and transparent solvents are needed, so that the photochemical reactions are carried out in hazardous solvents such as benzene, dichloromethane, acetonitrile or methanol. ${ }^{2}$

However, in the last decade there has been a significant effort made by photochemists to carry out reactions with more energy-saving and environmentally benign features. In this context, visible-light-induced organic reactions developed rapidly and have gained a considerable interest in both academia and industry. ${ }^{3}$ Light-emitting diodes (LEDs) 
are successfully used with this purpose, as they are able to provide high-intensity visible light over a short wavelength range. Sources of this kind have the advantages of economy and long life. LEDs emitting in the UVA, down to $320-310 \mathrm{~nm}$ are also available, although their emission is much less intense, and they are more expensive. In the same way, solar light as a more sustainable energy source has been evaluated in photochemical reactions, opening the possibility to explore a greener process. ${ }^{4}$

Despite continuous progress and technical developments in the use of photochemistry, its application in industry has been limited by the scalability of photochemical processes, which require powerful light sources and reactors of special dimensions and shapes to produce large amounts of chemicals per unit of time. Over the last decade, the use of continuous-flow chemistry has emerged as a powerful technique for photochemical applications, making it possible to overcome the issues associated with batch reactors. ${ }^{5}$ Although in the last years a series of reviews have been published showing the advantages of flow photochemistry in synthetic application, ${ }^{6,7,8}$ the aim of this review is the development of flow photochemistry as applied to compounds of pharmaceutical interest. Furthermore, different scale-up strategies of continuous multistep synthesis will be discussed, showing future perspectives in flow chemistry.

\section{Flow Photochemistry}

\section{The Advantages of Continuous-Flow Processes ${ }^{9,10}$}

With the development of flow chemistry, Organic Photochemistry has gained value for the synthetic community and industry due to its interesting applications in milder and greener conditions. One important problem for photochemical reactions that use continuous-flow processes is the ability to scaling it up easily using different reactor volumes or parallel multi-reactors (numbering-up). ${ }^{11,12,13}$ Scalability is directly related 
to an efficient and uniform irradiation of the reaction mixture, which can be obtained according to the well-known Bouguer-Lambert-Beer law, by using narrow channels in the microreactor. Furthermore, with adequate use of refractors or miniaturized light sources, the photon flux (number of photons observed per unit time) is more efficient, resulting in an acceleration of the photochemical reaction (Figure 1). For this reason, a reduction of byproducts formation from over-irradiation could be observed, with a consequent increment of productivity. Additionally, due to the size of the microreactor, faster mixing is achieved, enhancing the mass- and heat-transport thus providing a high reproducibility of the photochemical conditions and improving the selectivity of the reaction. ${ }^{14}$

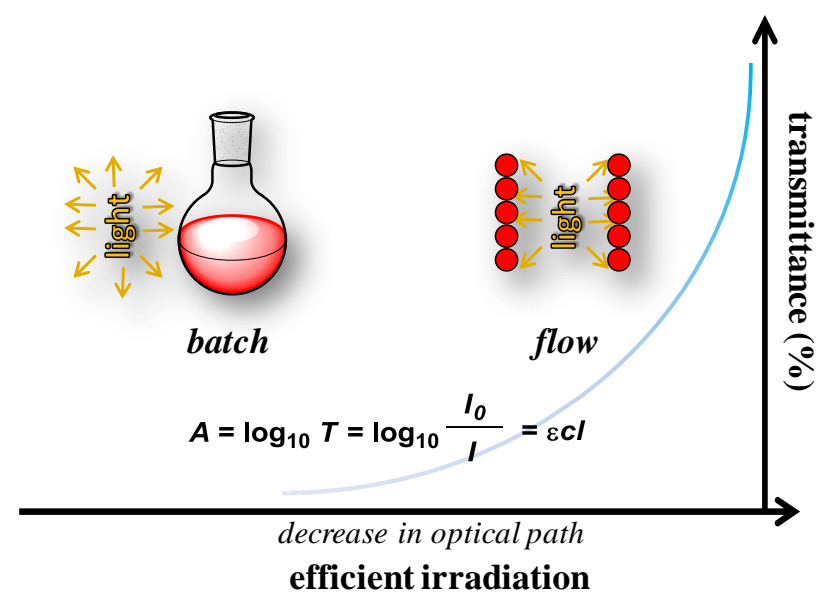

Figure 1. Transmission of light as a function of the optical path according to the Bouguer-Lambert-Beer correlation in a photochemical process in batch vs. flow.

On the other hand, one interesting advantage of flow chemistry is the fact that multiphase reactions can be carried out in a simple way and under safe conditions. Specifically, the interfacial area is maximized in microreactors because of their size, leading to an efficient mass transfer between the two phases (Figure 2). Segmented flow obtained by liquid slug and elongated bubbles from the immiscible phase has been 
successfully used in different photochemical processes. Particularly, photo-oxygenation reaction has been improved by using a slug flow with oxygen or air as immiscible phase. ${ }^{15}$ Additionally, an improvement in the efficiency of photocycloaddition has been observed in slug flow conditions, with water as immiscible phase. ${ }^{16}$
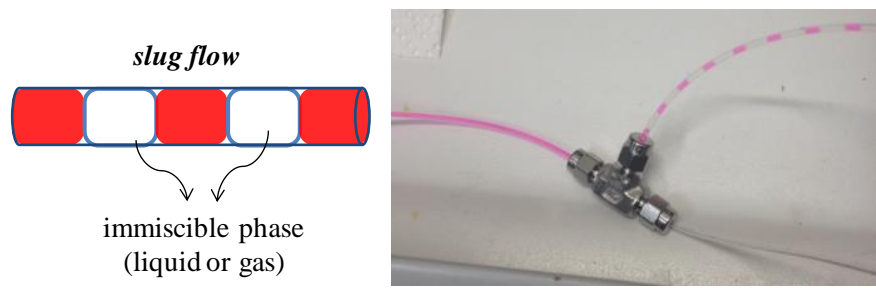

Figure 2. a) Schematic representation of a slug flow for multiphase reactions. b) Real picture of a slug flow with colorized water and air as immiscible phase for a photooxygenation reaction. Reprinted with permission from ref 15. Copyright 2015 John Wiley and Sons.

\section{Continuous-Flow Technology}

From their beginning, flow photochemical reactions were carried out using simple reactor components that combined pumping systems with different reactor bodies and available light sources. For the academic, an interesting way of improving photochemical reaction and of continuing displaying its synthetic potential is the use of available and cheap equipment to access to flow-photoreactor. Even though at present commercial flow photoreactors are available such as Vapourtec UV-150, ${ }^{17}$ Corning Advanced-Flow G1 Photo Reactor or Corning Advanced-Flow Lab Photo Reactor, ${ }^{18}$ Heraeus Noblelight, ${ }^{19}$ many efforts are still made to build simple "in-house" designs. One of the most commonly used flow photoreactors consists of flexible fluorinated ethylene propylene (FEP) or polytetrafluoroethylene (PTFE) tubes wrapped around a glass cylinder and irradiated by a lamp at its center, which means that it can easily be constructed, using cheap and readily available materials. ${ }^{20}$ In this way, Noël and co- 
workers have described a protocol for building a compact, inexpensive modular photomicroreactor using commercially available parts, which can be used in a wide variety of homogeneous visible light photocatalysis or UV-mediated reactions (Figure $3)^{21}$

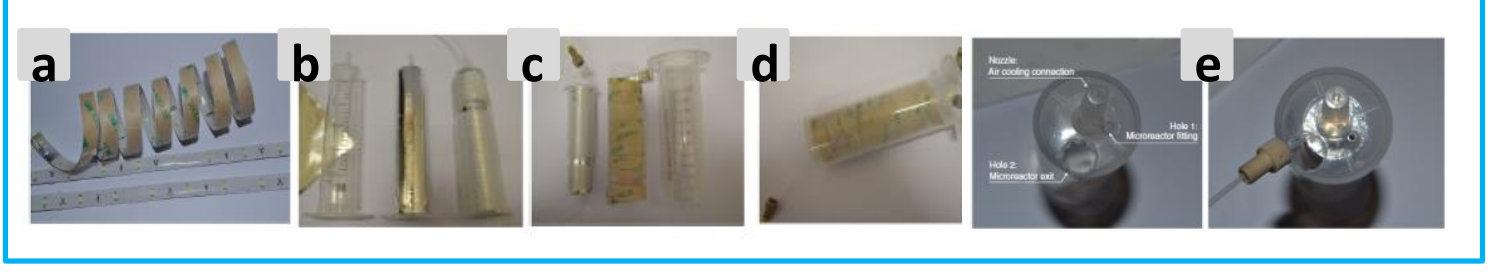

Figure 3. Different parts and sequence for constructing the "in-house" photomicroreactor. a) LED strips. b) Refractive tape around a 20-ml syringe, followed by the PFA microtubing coiled around the syringe (microreactor). c) Large syringe as LED place holder. d) LED array and microreactor positioned inside the place holder (finished photomicroreactor). e) Holes drilled in the larger-diameter syringe used to hold the photomicroreactor in place to fit the exit of the microreactor. The nozzle provides air cooling. Reprinted with permission from ref 21. Copyright (2016) the American Chemical Society.

Another particular photoreactor design was implemented by Pergantis, Vassilikogiannakis and co-workers, base on pneumatically generated aerosols to undertake bi-phasic photooxygenations. ${ }^{22}$ This particular reactor works by nebulizing a photo-oxidation solution inside a cylindrical chamber, mimicking the earth's atmosphere clouds. A low energy LED strip surrounds the chamber and either oxygen or air can be used in excess for efficient nebulization of the reactant solutions. One of the photo-oxygenation reaction tested was the ene reaction of $\beta$-citronellol, carried out in the presence of $0.8 \mathrm{~mol} \%$ of rose Bengal as a phtosensitizer, with the ability to 
maximize the surface area to volume ratio between the two phases, and consequently an exceptionally high productivities is obtained.

More recently, Booker-Milburn's group has developed a new flow photoreactor where photochemical synthesis can be carried out on a kilogram scale over a $24 \mathrm{~h}$ processing period. ${ }^{23}$ In addition, a 10-fold reduction of reaction time was achieved compared with a FEP reactor. The modular reactor consists of an array of a series linked of quartz tubes axially arranged around a high power light source (e.g., Hg-lamp or LEDs), with size not much larger than a rotary-evaporator condenser. In this way, the reaction solution enters through an end-cap, flow through one tube into the opposite end-cap, and then back through the adjacent tube in the opposite direction (Figure 4a).

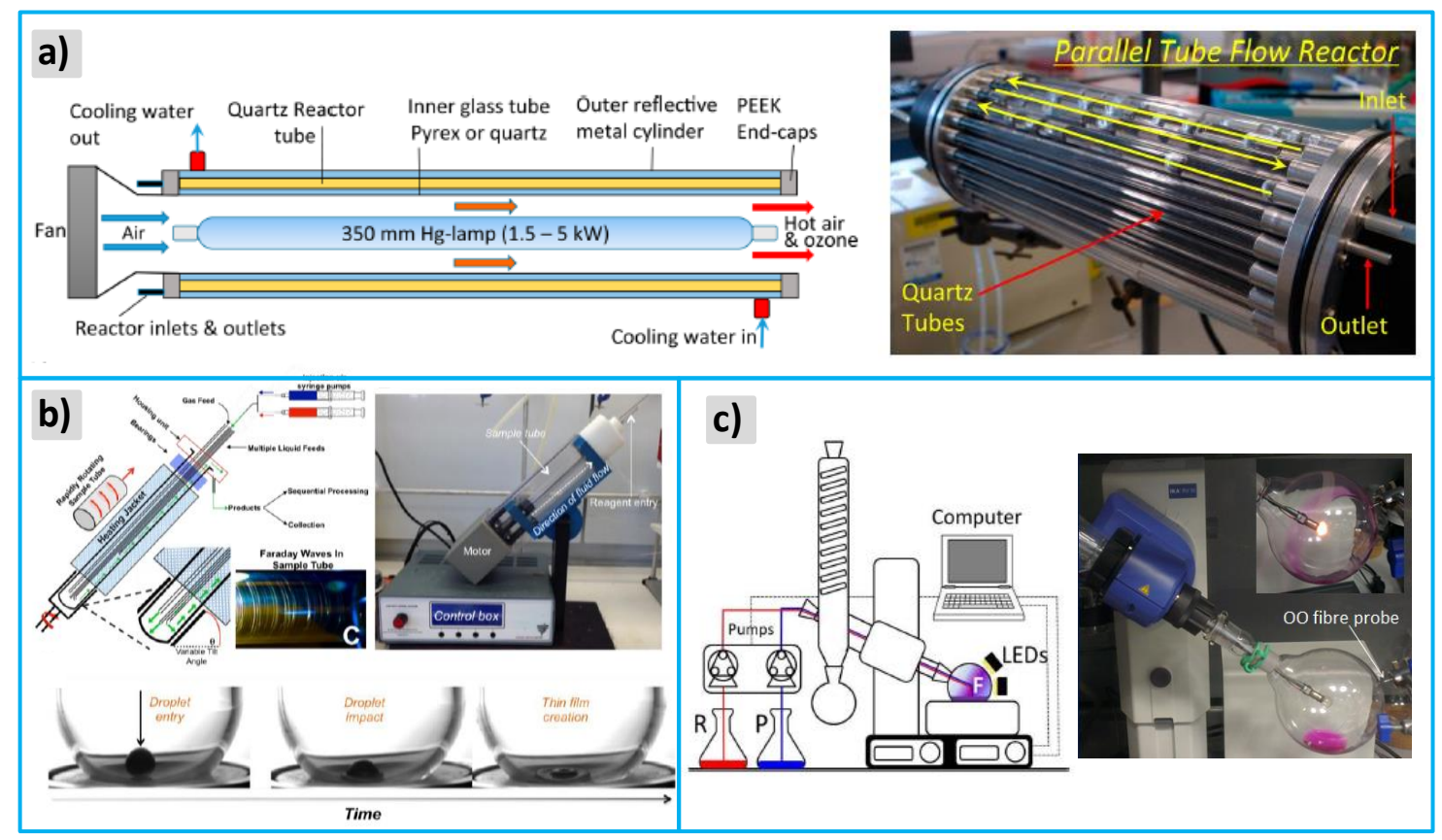

Figure 4. a) Schematic representation and real picture of an immersion-cooled parallel tube flow reactor. Reprinted with permission from ref 23. Copyright (2016) the American Chemical Society. b) Schematic and real picture of the vortex fluidic device (VFD). The image highlights droplet entry into the reactor, its subsequent impact, and the creation of the thin film. Reprinted with permission from ref 24. Copyright (2017) the John Wiley and Sons. c) Schematic representation and real picture of the reactor 
based on a slightly modified lab rotary evaporator. Reprinted with permission from ref 25. Copyright (2016) the American Chemical Society.

Another application of flow photochemistry is the use of thin films processing of the reaction medium. A thin film can be generated using a vortex fluidic device (VFD). When a fluid enters the vortex system, the droplet impacts the hemisphere of the rotating tube to form a thin film. The film's effect allows a larger surface-to-volume ratio, resulting in a more efficient and homogenous irradiation, rapid heat transfer and uniform mixing of reagents. In this case, the rotational speed and tilt angle of the tube are what define the efficiency of the reaction. This development of VFD has been successfully used by Raston's group in a variety of synthetic applications ${ }^{24}$ and has been extended to photochemistry (Figure $4 b){ }^{25}$

Applying the principle of thin film in an elegant way, George and co-worker have been able to use a conventional rotary evaporator in photochemical reactions. Additionally, a rotary evaporator can be adapted in a simple way for use as flow reactor by the introduction of two thin PTFE tubes through the rotating neck to allow a solution to be pumped in and out of the reactor. A vacuum pump is not necessary and LEDs or any other light sources can be placed around the flask. The reactor can be operated in semicontinuous mode, showing that flow photochemistry is nowadays accessible to any laboratory group (Figure $4 \mathrm{c}){ }^{26}$

Building on the pioneering work of Giacomo Ciamician, who was one of the first to work with organic chemistry and plant chemistry evaluating photochemical reaction under sunlight, ${ }^{27}$ Noël, Debije and co-workers have developed a tree leaf-inspired photomicroreactor which may be used in the future to promote solar photochemical synthesis (Figure 5). ${ }^{28}$ Many things have been taken into consideration in order to 
develop an efficient device in which sunlight reactions can be carried out. The concept of "luminescent solar concentrator" (LSC) was used. LSC captures solar light through a dispersed luminophore (such as a fluorescent dye) in a glass or polymer support. In addition, microreactor technology was conveniently used to provide a high surface-tovolume ratio of solar light and to improve its large-scale application. LSC is a polymeric material constituted by Lumogen F Red 305 (LR 305), a perylene bisimidebased dye. It has an emission profile that matches the absorption spectrum of the methylene blue (MB) photocatalyst used in the reaction system. The luminescentconcentrated photon flux into the microchannels is more intense than the solar light and results in an efficient [4+2] cycloaddition between singlet oxygen and 9,10diphenylanthracene.

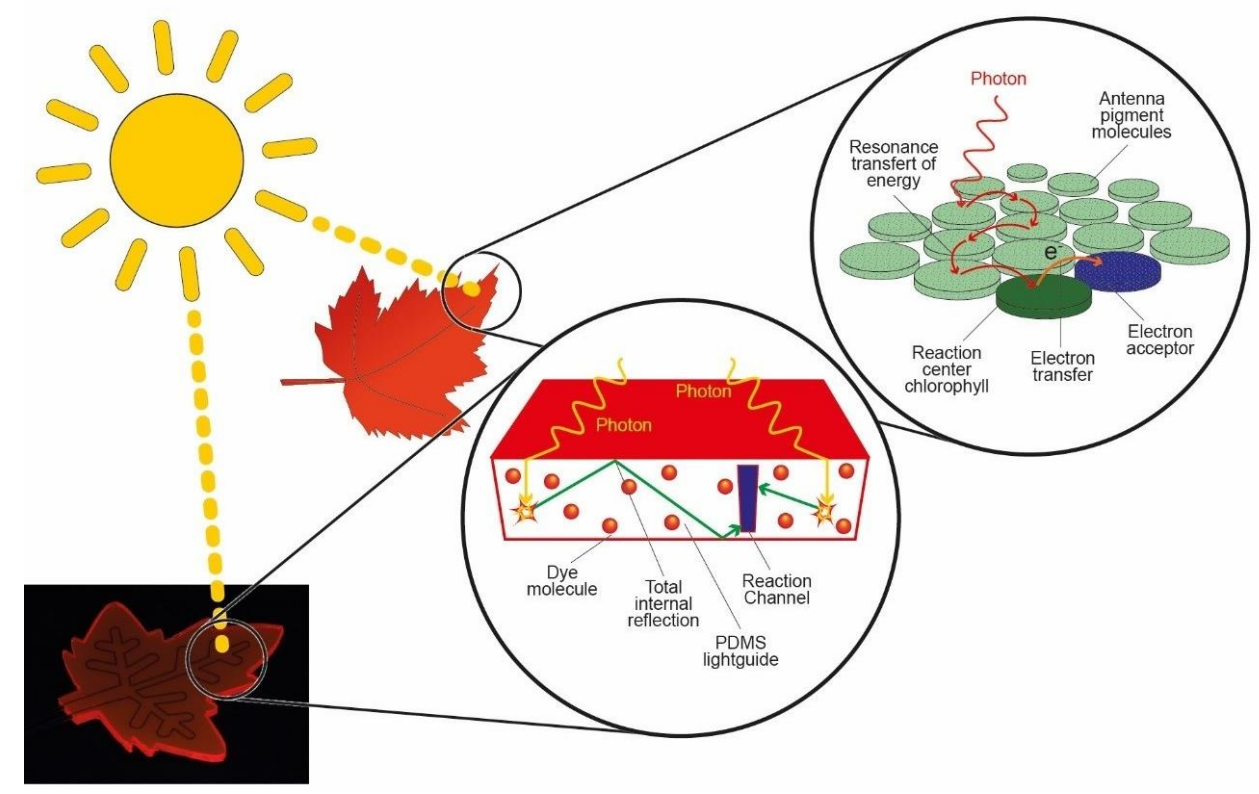

Figure 5. Leaf-inspired photomicroreactor. A leaf photosystem uses solar energy harvesting by means of antenna pigment molecules and a photon transfer process to carry out the reaction. Similarly, in a LSC microfluidic reactor chip the photons are absorbed by the fluorescent molecules and are re-emitted toward the edge of the device where a photosensitizer is attached to carry out the photochemical reaction. Reprinted with permission from ref 28. Copyright 2017 John Wiley and Sons. 
It is clear that a control system with different parameters of photochemical reaction is needed to increase the efficiency and productivity of the reaction. Nowadays, many research groups are working on the use of automation platforms, assembling on-line analytical techniques or control modules to optimize a photochemical reaction in a very simple way, and in just few minutes. Following its work; Nöel's group has employed process control technologies which act in real-time to automatically adjust the residence time to the solar irradiance variation in the LSC photomicroreactor. ${ }^{29}$ Additionally, they development a fully automated continuous-flow platform suitable for luminescence screening conditions and Stern-Volmer analysis applied to a photoredox catalysis, in which a spectrophotometer and light source are controlled by software and a HPLC pumps regulate the stream to the flow cuvette. ${ }^{30}$

In the same way, Jensen and co-workers were able to screen and optimization (residence time, light source power, temperature) a series of visible-light photoredox liquid or gasliquid catalysis in a $15 \mu \mathrm{L}$ scale, using an automated microfluidic platform. An oscillatory flow is controlled by a computer, enabling a flexible residence time keeping mixing and heat transfer efficiently. ${ }^{31,32}$

\section{Safer Photo-oxygenations}

Molecular oxygen is a green oxidant and has attractive features: it is inexpensive, environmentally benign, and its reactions involve an atom economy. However, one of the challenges for chemists or the industry is to use oxygen as reagent in large scale under controlled and safe conditions. Generally, oxidations reactions which use oxygen or air are exothermic, and the heat generated can be difficult to dissipate. In conventional batch reactors, gas-liquid reaction using organic solvent at a limiting 
oxygen concentration (LOC) has to be applied to avoid spontaneous ignition. Furthermore, temperature and pressure are difficult to control, which results in a low selectivity and incomplete reaction.

With the development of continuous flow technology, aerobic oxidation or even the use of oxygen as reagent has become possible without safety hazards. High temperatures and pressures are easily controlled through the use of high pressure resistant tubing and small reaction volumes that can be rapidly cooled. Moreover, a gas-liquid oxidation in flow features an excellent mass and heat transfer, minimizing the possibility of explosion when flammable solvent are used. ${ }^{33}$ Heterogeneous reactions can be carried out using a segmented (slug) flow which allows a more efficient mixing, with improved mass transfer between the phases (Figure 2). ${ }^{34}$

Specifically, photo-oxygenations involve the participation of singlet oxygen $\left({ }^{1} \mathrm{O}_{2}\right)$ as reactive species which can be formed in situ under photosensitizer oxygen conditions. Singlet oxygen has a high selectivity, and is in fact a very reactive electrophilic species, able to react with unsaturated substrates leading to the formation of endoperoxides, dioxetanes or allylic hydroperoxides. It can also react with sulphur, selenium, phosphorus and nitrogen compounds. Photo-oxygenation represents a powerful strategy to obtain oxy functionalized compounds under mild experimental conditions, becoming a valuable tool for the synthesis of natural products. ${ }^{35,36}$

Another reactive species from molecular oxygen is the superoxide radical anion $\left(\mathrm{O}_{2}{ }^{-}\right)$ which can be formed by a Singlet Electron Transfer (SET) process from a reduced photocatalyst in an air or oxygen atmosphere. These highly reactive species are capable of oxidizing different substrates such as aryl boronic acids or phenols, and are involved in a proton abstraction pathway. ${ }^{2}$ 
Many of the intermediate products derived from photo-oxygenation are thermally unstable or are explosive peroxides. Furthermore, the concentration of reactive species from oxygen is very important but due to high reactivity an over-oxidation of products can be observed. For all of these, the use of continuous-flow chemistry has a key advantage for photochemical transformations, allowing as it does a precise control of the parameters of the reaction, and enabling work to be done under safer conditions. ${ }^{37,38}$ As an offshoot from this technology, chemists and engineers have developed a multitude of flow reactors to generate reactive species from oxygen, carrying out interesting photochemical applications. In this context, Dreesen, Heinrichs, Monbaliu et. al. used a compact commercial glass mesofluidic module (Corning Lab Reactor) to carry out a scalable photo-oxygenation of methionine under mild conditions, improving chemo-selective and atom-economic, and implementing a safer and sustainable process. ${ }^{39}$ The photo-reactor design combines high mixing with an efficient irradiation. With an integrated static mixers and sandwiched in a high capacity heat-exchanger, the multiphase mass transfer could be optimized and LED panels were put on both sides of the fluidic device. Additionally, the flow-through module was equipped with an in- and offline NMR to analyze the reactor effluent. Under the best conditions a $1 \mathrm{~mol} \mathrm{day}^{-1}$ of methionine sulfoxide was obtained using a very low loading of photocatalyst $(0.1 \%$ Rose Bengal) at room temperature with white light irradiation (4000K) and just 0.1 equivalent of oxygen.

In the same way, Oelgemöller and coworker demonstrated the utility of a commercially available falling film microreactor (IMM, Mainz) by applying it in a photo-oxygenation reaction. ${ }^{40}$ The miniaturization of flow devices has the advantage of setting the parameters of the reactions under precise control and allowing the reactions' progress to be monitored on-line. Because of that, microfluidic devices can be utilized for the 
optimization of reactions which involved the use of hazardous reagents. Particularly, the photo-oxygenation of $\alpha$-terpinene was studied, evaluating reproducibility, oxygen sources, solvent, substrate and sensitizer concentration, oxygen flow rate, temperature and light sources. Photo-oxygenation takes place in the interface between the liquid and the gaseous layer, which is obtained in a thin film of the liquid reaction generated by gravity and counter-currently to the gas phase. Using Rose Bengal (RB) as ${ }^{1} \mathrm{O}_{2^{-}}$ photosensitizer, endoperoxide ascaridole, a natural molecule with important bioactivities, was obtained with a yield of up to $89 \%$ yield with a productivity of 2.5-3.2 mol L ${ }^{-1} \mathrm{~h}^{-1}$ (Scheme 1).

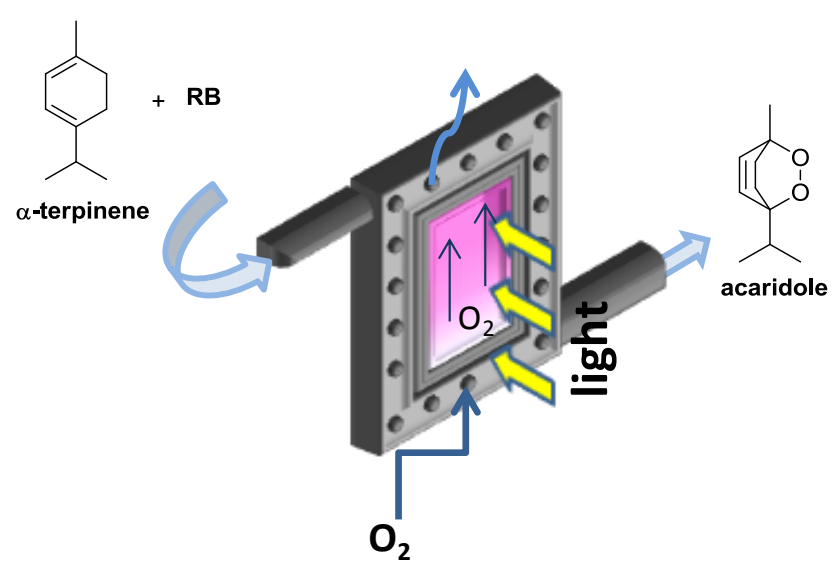

Scheme 1. Photo-oxygenation of $\alpha$-terpinene in a falling film microreactor.

Another recent study involves the use of a vortex reactor to carry out continuous photooxygenations. In this case a rapid rotation generates a Taylor vortices flow presenting certain advantages for gas/liquid heterogeneous reactions. ${ }^{41}$ The high mass transfer between both layers allows a more efficient dissolution of gases, owing to which air from the lab can be used instead of pressurized oxygen. The versatile application of a vortex reactor could be demonstrated in different photochemical reactions using ${ }^{1} \mathrm{O}_{2}$ or $\mathrm{O}_{2}{ }^{--}$as reactive species. Phenyl boronic acid was selectively photo-oxidized to the corresponding phenol; additionally, photo-oxygenations of furfuryl alcohol and $\alpha$ - 
terpinene were carried out with a high yield and efficiency. In the case of $\alpha$-terpinene with a rotation speed of $4000 \mathrm{rpm}$ a $91 \%$ yield of endoperoxide ascaridole was obtained with a productivity of $2.73 \mathrm{mmol} \mathrm{h}^{-1}$.

Another interesting application of photo-oxidation in a segmented flow regime was recently developed by Nöel and co-workers. Activated and unactivated $\mathrm{C}\left(\mathrm{sp}^{3}\right)-\mathrm{H}$ bonds were selectively oxidized via decatungstate-photocatalysis (TBADT) with yields ranging from (41-91\%) in a short reaction time (45 $\mathrm{min})$. This methodology was extended to the oxidation of biologically active compounds, such as (-)-ambroxide, pregnenolone acetate, (+)-sclareolide, and artemisinin showing its versatility (Scheme $2) .42$
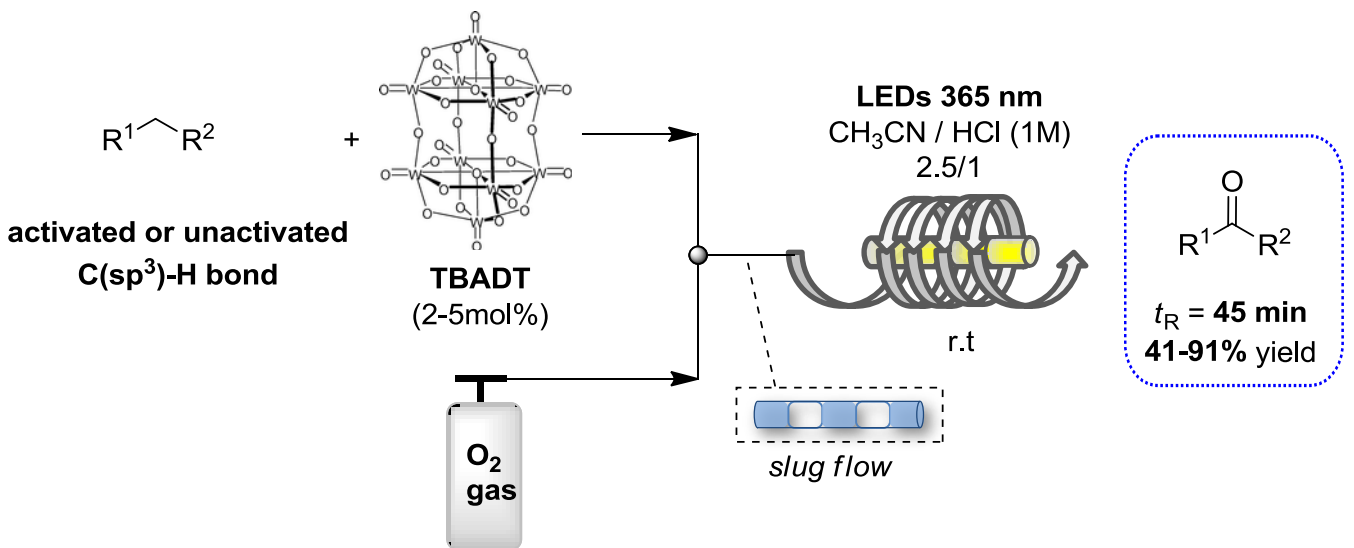

Scheme 2. $\mathrm{C}\left(\mathrm{sp}^{3}\right)-\mathrm{H}$ oxidation enabled by decatungstate photocatalysis (TBADT) in slug flow.

\section{Continuous flow photochemistry for the synthesis of compounds of pharmaceutical interest}

The strength of the continuous flow process can be demonstrated by its application to the preparation of fine chemicals such as natural products, but additionally through the synthesis of APIs. Puglisi, Benaglia and co-worker reviewed the advances in continuous multistep applied to APIs synthesis. ${ }^{43}$ More recently, Kappe and Dallinger have 
critically reviewed the use of continuous processes in the synthesis of APIs, analyzing their impact in terms of sustainability. ${ }^{44}$

Taking into account that the synthesis of natural products or target molecules which display biological activity is an essential part of drug discovery, and that the APIs generally have an inherent complexity associated with their molecular framework, the development of safer and more sustainable synthesis protocols could have a high impact on the pharma industry. In this context, the combination of flow and photochemistry is an excellent approach which, over recent years, has been employed successfully. In order to provide new strategies for future transformations applying flow photochemistry, selected examples of continuous photochemical transformation of natural products or molecules target synthesis from the last three years are presented in this section.

\section{Photocycloaddition Reaction}

One of the photochemical reactions most widely used for natural product access is [2+2] photocycloaddition, which allows the obtaining cyclobutane rings in a simple way. ${ }^{45}$ Beeler and co-workers successfully synthesized $\delta$ and $\beta$-truxinate by means of a headto-head photodimerization from methyl cinnamate. ${ }^{46}$ These cyclobutane derivatives and other related molecules, such as the natural products piperarborenine and incarvillateine, exhibit antineuroinflammatory activity and act as selective glucose and lipid homeostasis PPAR $\gamma$, becoming an attractive drug target for therapy of type 2 diabetes mellitus and dyslipidemia. ${ }^{47,48,49}$ Using a cone photo-flow reactor they could improve the $[2+2]$ photocycloaddition increasing yields in a shorter time. Furthermore, when bis(thiourea) was used, an increment in the yield and diasteroselectivity was observed. This can be attributed to an organo-photocatalysis effect (Scheme 3). 

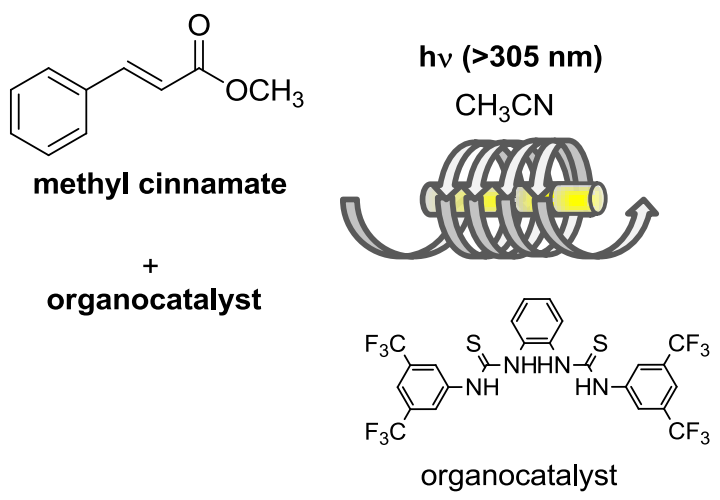

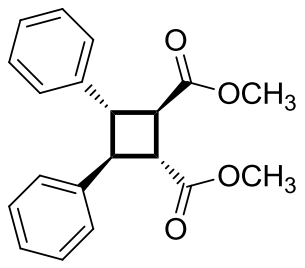

cyclobutane derivate $t_{\mathrm{r}}=\mathbf{8} \mathbf{h}$ $60 \%$ isolated yield

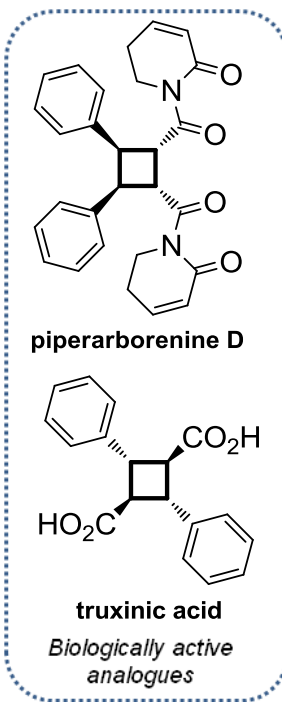

Scheme 3: Synthesis of $\delta$ and $\beta$-truxinate and analogues by a photocycloaddition $[2+2]$

In the same way, Urriolabeitia, Gomez, Cativiela et al., stereoselectively synthesized $\varepsilon$ isomers of dimethyl esters of 1,3-diaminotruxillic acid in three steps,${ }^{50}$ one of which involves a [2+2] photocycloaddition in flow as a key step. The reaction was carried out very efficiently by using a LED light source, obtaining quantitative yields in very short reaction times (5-20 minutes). This synthesis has the particularity that in the first step an ortho-palladation of oxazolone is carried out resulting in the formation of dimeric ortho-palladated complexes with visible light absorption. In addition, their rigid clamshell structure locates the two $\mathrm{C}=\mathrm{C}$ bonds in close proximity, favoring their interaction for the [2+2] photocycloaddition and promoting stereoselectivity in the palladated truxillic acid product. Finally, the depalladation was induced with molecular hydrogen in methanol, obtaining the cyclobutane ring derivative (Scheme 4). 


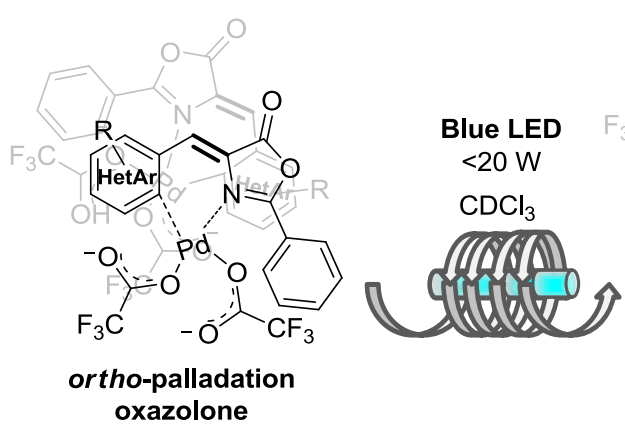

oxazolone

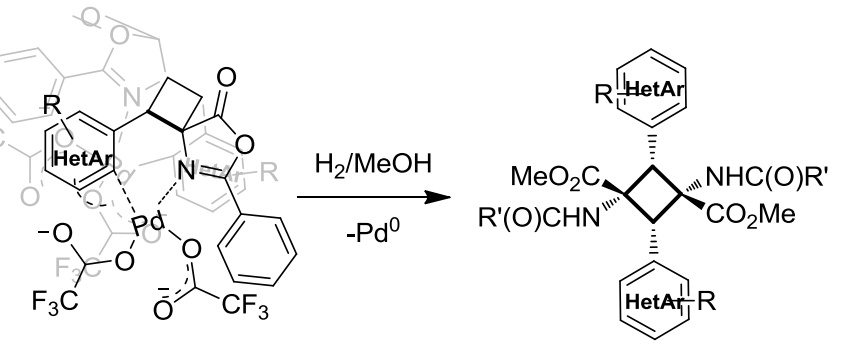

palladated truxillic acid

$t_{\mathrm{r}}=\mathbf{5}-\mathbf{2 0} \mathrm{min}$

$70-100 \%$ yield
$30 \min -2 \mathrm{~h}$

$31-41 \%$ yield one isomer

Scheme 4. [2+2]-Cycloaddition of ortho-palladated oxazolones.

Photocycloaddition is a well-established way of accessing strained ring scaffolds, which can be starting points for drug discovery. Moreover, using continuous flow technology it is possible to scale-up the reaction so as to generate enough material to carry out sequential steps in a complex synthesis. ${ }^{51}$ Pursuing these objectives, Booker-Milburn et al. described the obtaining of tricyclic aziridines in multigram quantities by flow photocycloaddition. These strained photochemical products can undergo different ringopening/annulation reactions to access a broad range of fused polyheterocycles. In this way, using aziridine as a starting point, they could synthesize the alkaloid ( \pm )-3demethoxyerythratidinone with a $68 \%$ yields after a 5 -step sequence (Scheme 5 ). ${ }^{52}$ This compound belongs to the Erythrina family of alkaloids, which has important pharmacological properties including sedative, hypotensive, CNS depressing, neuromuscular blocking and curare-like activities. ${ }^{53}$

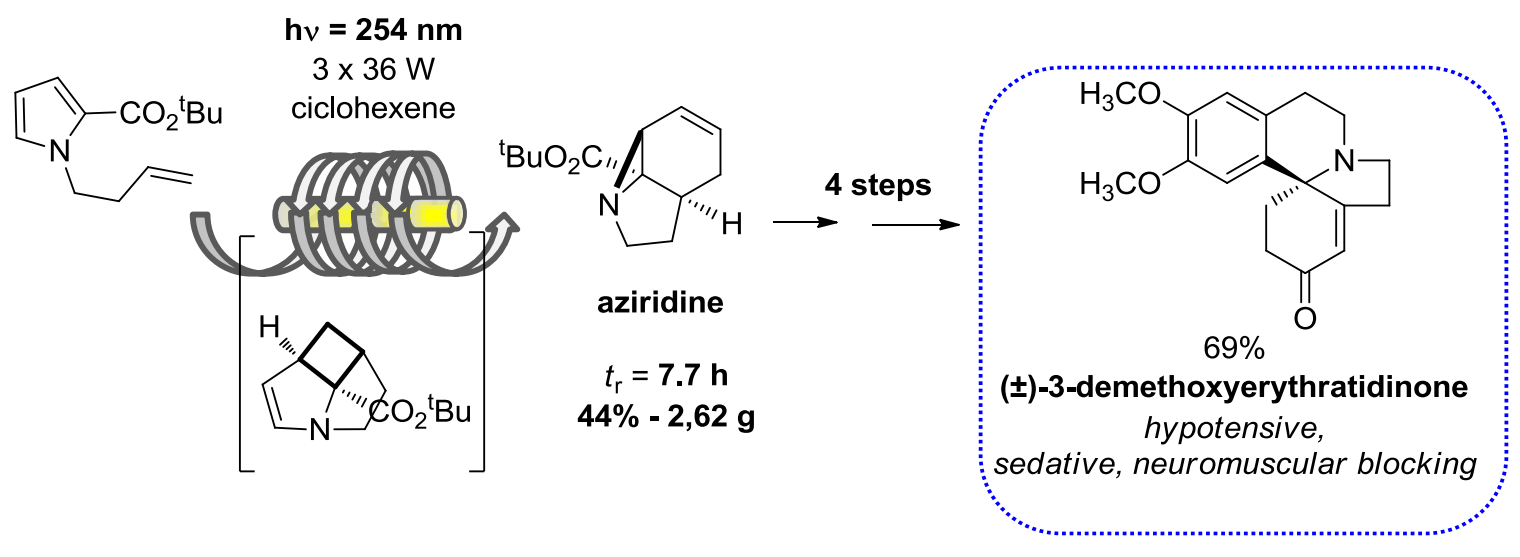


Scheme 5. Synthesis of the alkaloid ( \pm -3-demethoxyerythratidinone by $[2+2]$ photocycloaddition.

Following a similar approach, Ralph, Booker-Milburn et al. carried out the synthesis of (+)-Goniofufurone, a natural product found in Goniothalamus and also isolated from $G$. arvensis trees of the plant family Annonaceae, which exhibits promising antitumoral properties. ${ }^{54}$ Strategically, one step of the synthesis involves a Paternò-Büchi $[2+2]$ photocycloaddition in flow, which enables a very effective scaling up of the reaction (e.g., $150 \mathrm{~g} / 24 \mathrm{~h}, 97 \%$ isolated yield) in a short time. ${ }^{55}$ With multigram quantities of the key intermediary, the (+)-Goniofufurone could be obtained after five steps in $11.5 \%$ overall (Scheme 6).

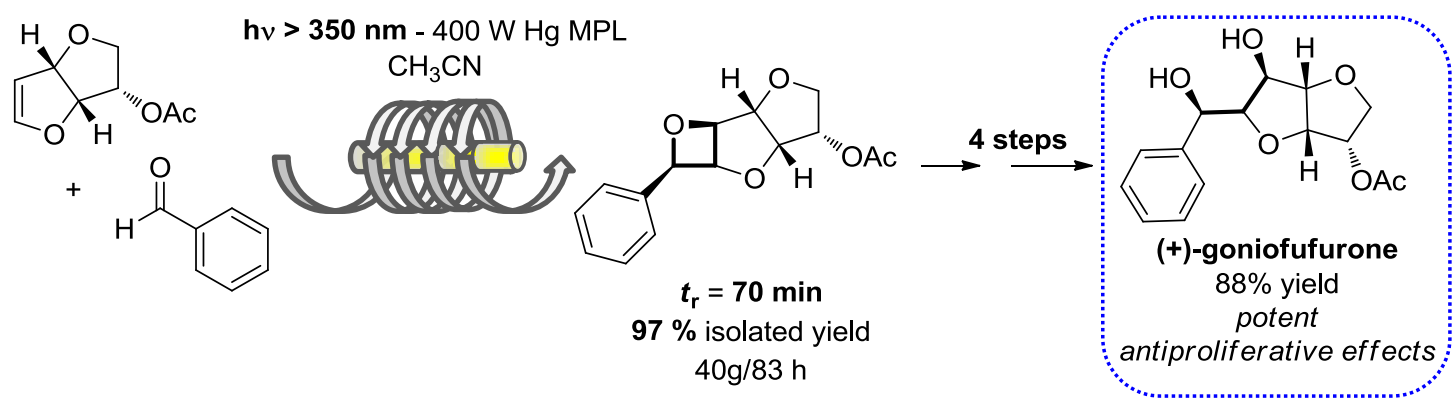

Scheme 6. Application of Paterno-Büchi cycloaddition to access (+)-goniofufurone.

There are other photocycloaddition processes which are often overlooked but which have been successfully implemented in natural product synthesis. One of them is $[3+2]$ cycloaddition that can involve an excited-state intramolecular proton transfer (ESIPT) of an adequate substrate to generate a reactive 1,3-dipole intermediate which can be trapped by a dipolarophile. Specifically, Beleer's research group carried out the construction of the aglains to generate tetrahydrocyclopenta[b]benzofuran core of the rocaglates and rocaglamides, using a [3+2] photocycloaddition in flow (Scheme 7). ${ }^{56}$ The Rocaglates are a complex secondary metabolite isolated from Aglaia genus plants. 
There are family members such as rocaglamide, silvestrol, rocaglaol, which exhibits potent cytotoxicity for a number of cancer cell lines. ${ }^{57}$ With a numbering-up approach, a multigram scale from ESIPT [3+2] photocycloaddition was developed to successfully generate aglain derivatives up to $12 \mathrm{~g}$ in $10 \mathrm{~h}$. Finally, the rocaglate skeleton was achieved after subsequent $\alpha$-ketol rearrangement and hydroxyl-directed reduction.
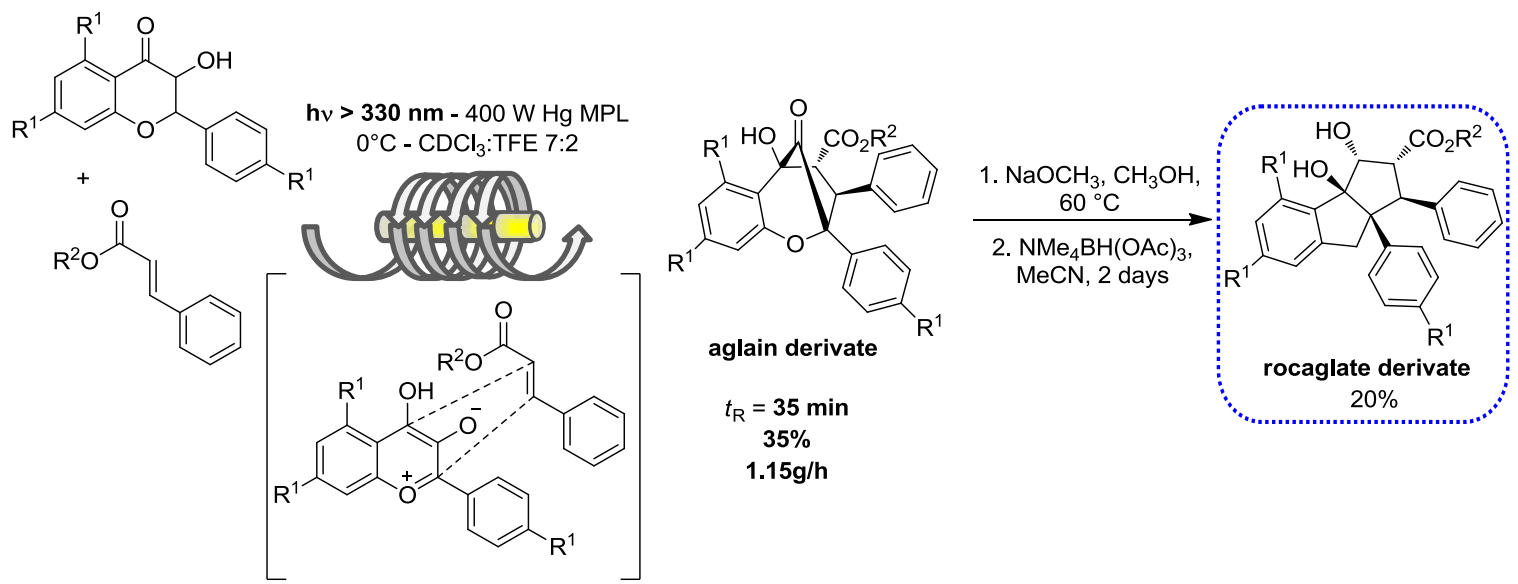

Scheme 7. [3+2] photocycloaddition to obtaining rocaglate derívate.

\section{Photocyclization Reaction}

Photocyclization is another kind of photo-induced reaction used to access heterocyclic skeletons of many natural products. Tranmer et al. have developed a continuous flow synthesis of substituted-6(5H)-phenanthridinones. ${ }^{58}$ The phenanthridinones family have been known to be inhibitor of Poly(ADP-ribose) polymerase (PARP) and are currently being used in clinical trials as potential cancer therapies. ${ }^{58}$ The method involves a twostep process where 2-halobenzanilide derivatives are generated from an aniline, followed by photocyclization to obtain a substituted-6(5H)-phenanthridinone in yields of up to $99 \%$. 


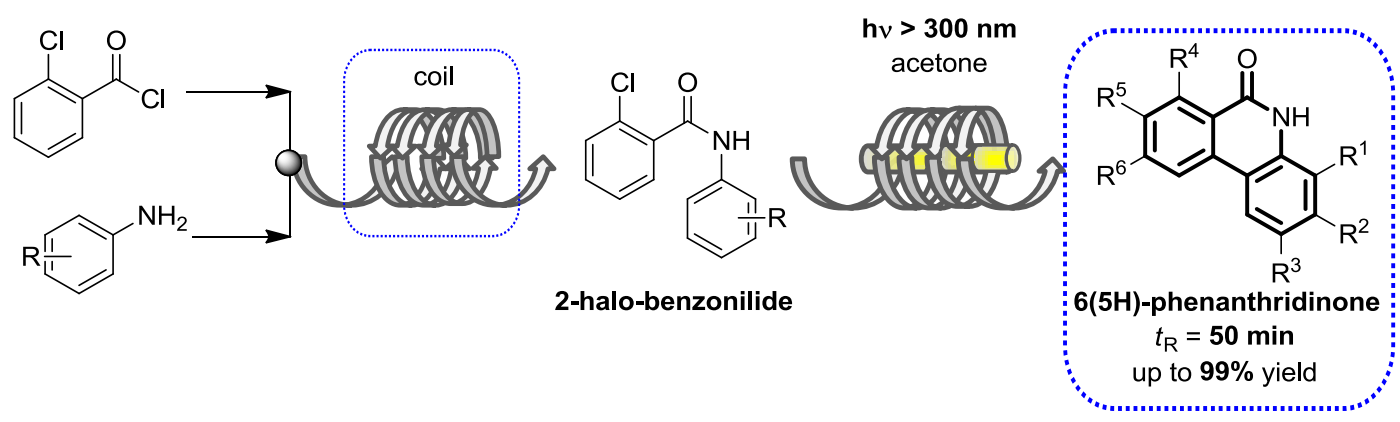

Scheme 8. Synthesis of substituted-6(5H)-phenanthridinones by photocyclization.

Koolman and co-worker carried out the synthesis of vabicaserin analogues by photocyclization reaction, using a mesoscale and a microfluidic flow photoreactor. $^{59}$ Vabicaserin is a polycyclic system with a 1,2,3,4-tetrahydroquinoline core, which has been clinically investigated as a $5-\mathrm{HT}_{2 \mathrm{C}}$ agonist for the treatment of schizophrenia. ${ }^{60}$ Furthermore, 1,2,3,4-tetrahydroquinoline are the substructure of many biologically active compounds and natural products, and as a consequence. ${ }^{61}$ Using photochemistry, Koolman's group could access 4'-dialkyl or spirocylic-substituted tetrahydroquinoline as part of a diazepino-annulated heterocycle. Particularly, 4,4'-disubstituted tetrahydroquinolines were obtained effectively using a mesoscale tube-based flow reactor in acetone as solvent and Pyrex as a filter. The Vabicaserin analogues could be synthesized in good yields after reduction with borane and subsequent Boc deprotection with trifluoroacetic acid. Furthermore, 4-spirosubstituted dihydroquinoline moiety was synthesized for the first time by photocyclization reaction in a microfluidic reactor with a $50 \%$ of isolated yield (Scheme 9). 


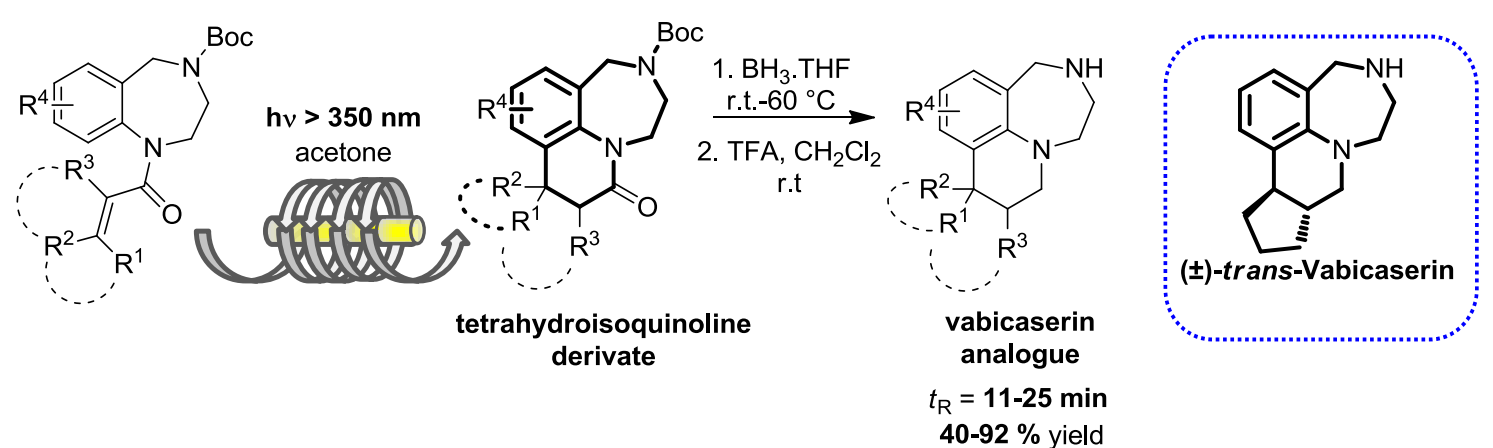

Scheme 9. Obtaining vabicaserin analogues through photocyclization reaction.

Czarnocki and Lisiecki have reported the use of photocyclization as a crucial step in the total synthesis of $(+)$-epigalcatin. ${ }^{62}$ The galcatin structure is present in many natural products with potential biological activity. Podophyllotoxin and semisynthetic derivatives are antiviral and cytotoxic compounds of clinical use and additionally are used as anticancer agents. ${ }^{63}$ The authors achieved the synthesis starting from commercially available and cheap materials including piperonal, 3,4dimethylbenzaldehyde, and diethyl succinate to obtain (+)-epigalcatin in a highly stereoselective way. Using L-prolinol as chiral auxiliary, an 8-membered ring was obtained via macrolactonization, setting the stage for the continuous flow photocyclization of the chiral atropisomeric 1,2-bisbenzylidenesuccinate amide ester with an isolated yield of $65 \%$. By chiral auxiliary removal and subsequent reductions, (+)-epigalcatin was achieved with an overall yield of 5\% (Scheme 10). 


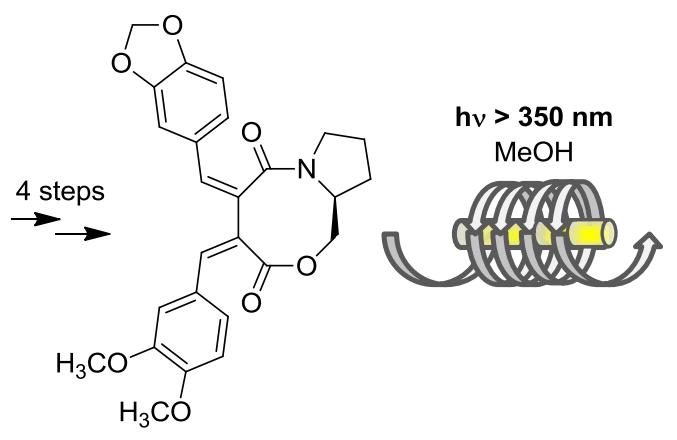

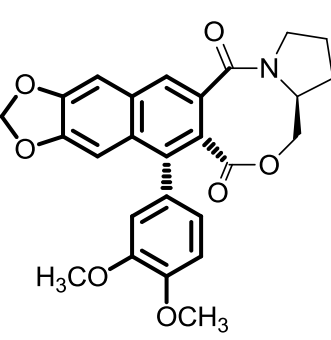

1,2-bisbenzylidenesuccinate amide ester $t_{\mathrm{R}}=15 \mathrm{~min}$ $65 \%$ yield

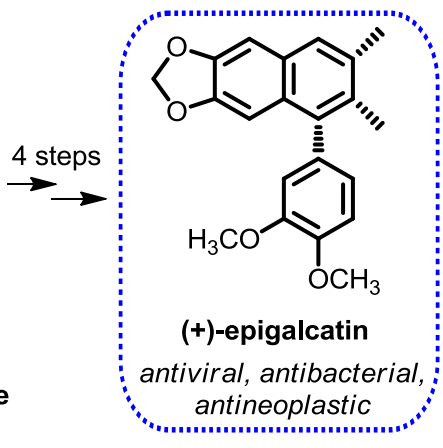

antiviral, antibacterial,
antineoplastic

Scheme 10. Total synthesis of (+)-epigalcatin employing photocyclization as a key strategic step.

Coombs and co-worker have developed a method to generate furan-fused cyclopentanones using a flow photochemical approach. ${ }^{64}$ Particularly, a photo-Nazarov reaction was employed as a key strategic step to access the corresponding heterocyclic motif which is present in the core structures of different alkaloid or flavonoid natural products with important biological activity. ${ }^{65}$ Starting with the 2-furyl or 2-thiophenyl enones and using a FEP tubing reactor, photocycloaddition was conducted using acetic acid (AcOH) or hexafluoroisopropanol (HFIP) as solvent, giving cyclic products in 4597\% yields, with exposure times of 3.4-6.8 min (Scheme 11).
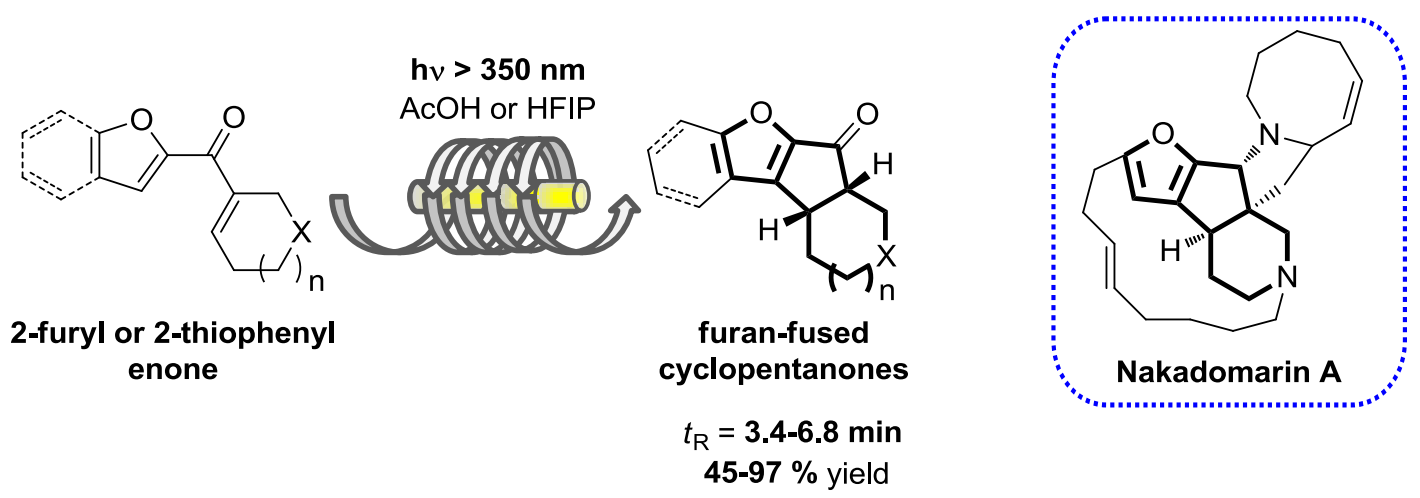

Scheme 11. Easy access to furan-fused cyclopentanones via photo-Nazarov reaction.

\section{Photoredox Catalysis Reaction}


Visible-light photoredox catalysis has emerged as an attractive strategy, as opposed to conventional methods to generate radical intermediates in an efficient and sustainable way. ${ }^{66}$ Starting with the fact that most organic molecules normally absorb in the UVvisible range $(254-350 \mathrm{~nm})$, the employment of photoredox catalysts which can be excited selectively in the visible-light range allows access to radicals and radical ions reactive species, by a Single Electron Transfer (SET) pathway, in mild and controlled conditions. In the last decade the use of visible-light photoredox catalysis has been applied in numerous organic transformations, and even in heterocyclic chemistry, ${ }^{67}$ and this catalysis has also been successfully applied to obtain known APIs. ${ }^{68}$ For this reason, it is not a surprising that flow photochemistry has been extended to photoredox catalysis in order to improve reaction times, yields and selectivity, as well as productivity.

Maintaining this approach, Collins and co-workers have extended their photochemical strategies for the construction of carbazoles to the use of visible-light photoredox and flow chemistry. In a previous study, the authors successfully synthesized carprofen analogues employing a continuous flow UV-reactor. ${ }^{69}$ Carprofen is a carbazole-based drug used in the treatment of pain in veterinary science. ${ }^{70}$ However, more recently, the visible-light-mediated synthesis of carbazoles with a $\mathrm{Cu}$-based sensitizer and the use of continuous-flow conditions resulted in an approximately 12-fold decrease in reaction time (Scheme 12a). ${ }^{71}$ In an alternative approach to carbazoles, aryl azides were used as a photochemical reagent allowing access to more complex heterocycles under UV-LED irradiation in a continuous flow reactor. ${ }^{72}$ Specifically, the anti-inflamatory Carprofen could be obtained with a residence time of $4 \mathrm{~h}$ with a $64 \%$ of isolated yield (Scheme $12 b)$. 

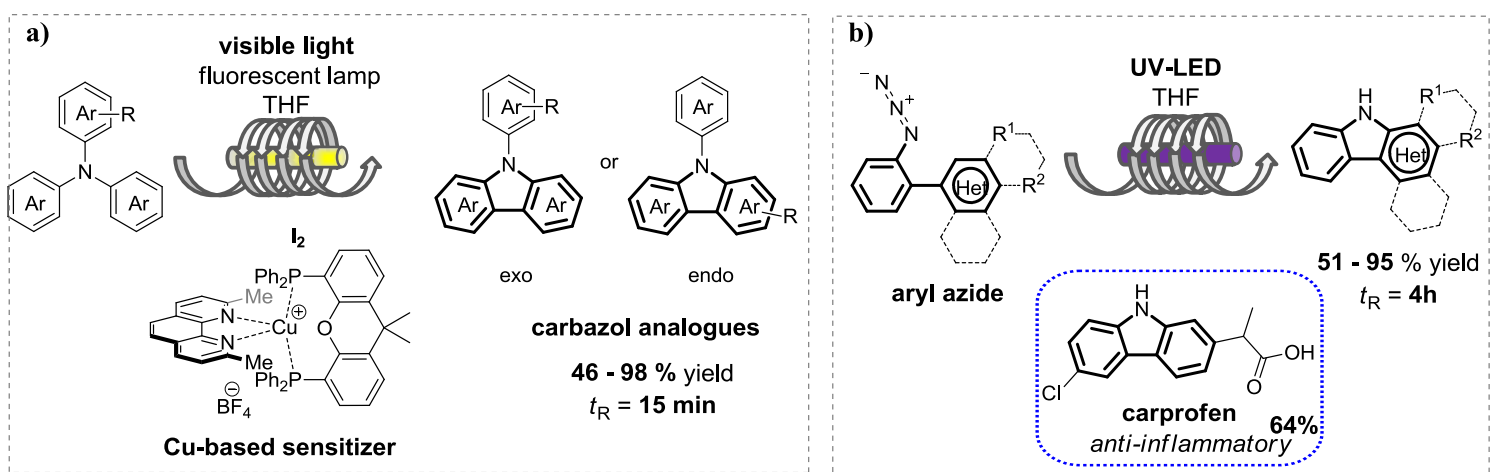

Scheme 12. Synthesis of carbazoles derivatives. a) Using photoredox catalysis with a

$\mathrm{Cu}$-based sensitizer. b) Using aryl azides as photochemical reagent.

In another synthetic strategy for building heterocycles, Stephenson and co-workers reported coupling of tertiary alkyl bromomalonate with electron-rich arenes, such asindole, thiopene or pyrrol, via visible-light protoredox catalysis. ${ }^{73}$ Furthermore, quaternary carbon centers were efficiently prepared in high yields and could be scaled up in a flow photoreactor. Taking into account that substituted malonate esters are key intermediates in the biologically active molecule synthesis, and have themselves shown antimycrobacterial properties, using this photochemical method it is possible to access interesting molecules such as bioactive thiophene malonate derivative, with therapeutic potential in cardiovascular and neurological diseases. The bioactive thiophene malonate derivative is an ion-channel modulating agent and it is known to modulate $\mathrm{Ca}^{2+}$ activated $\mathrm{K}^{+}$channels with subtypes SK/IK/BK (Scheme 13). ${ }^{74}$

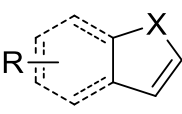

arene

$\mathrm{X}=\mathrm{O}, \mathrm{N}, \mathrm{S}$

$+$<smiles>[R]C([R])(Br)C(=O)OC</smiles>

visible light

blue LEDs

MeCN - r.t.

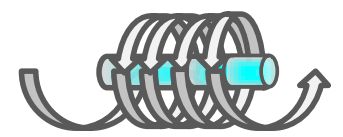

$\operatorname{Ir}(\mathrm{ppy}) 3$

2,6-lutidine

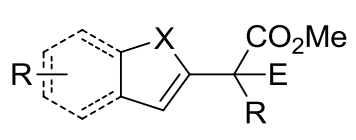

$t_{\mathrm{R}}=\mathbf{4 0} \min$

$78 \%$ yield

alkyl bromomalonate

Scheme 13. Visible light photoredox-mediated coupling of tertiary alkyl bromomalonate with electron-rich arenes. 
Chen and co-workers have implemented a strategy by photoredox catalysis to generate tricyclo-1,4-benzoxazines in continuous flow. ${ }^{75}$ Numerous pharmacological compounds have benzoxazines as scaffolds in their structure, conferring important bio-activities such as antitumor, anti-inflammatory, antihypertensive, neuroprotective as well as antithrombotic. ${ }^{76}$ Additionally, the same group has observed a potent anticancer activity in tricyclo-1,4-benzoxazine derivatives. Correspondingly, they reported a one-pot synthesis of benzoxazines starting with 3-subtituted indoles in the presence of methylene blue (MB) as a photocatalyst to generate singlet oxygen, followed by a rearrangement induced by $p$-toluenesulfonic acid $(\mathrm{TsOH})$. Using white LEDs light, after $1 \mathrm{~h}$ in a continuous flow reactor, a family of 1,4-bezoxazines were obtained in yields ranging from 52-99\% (scheme 14).

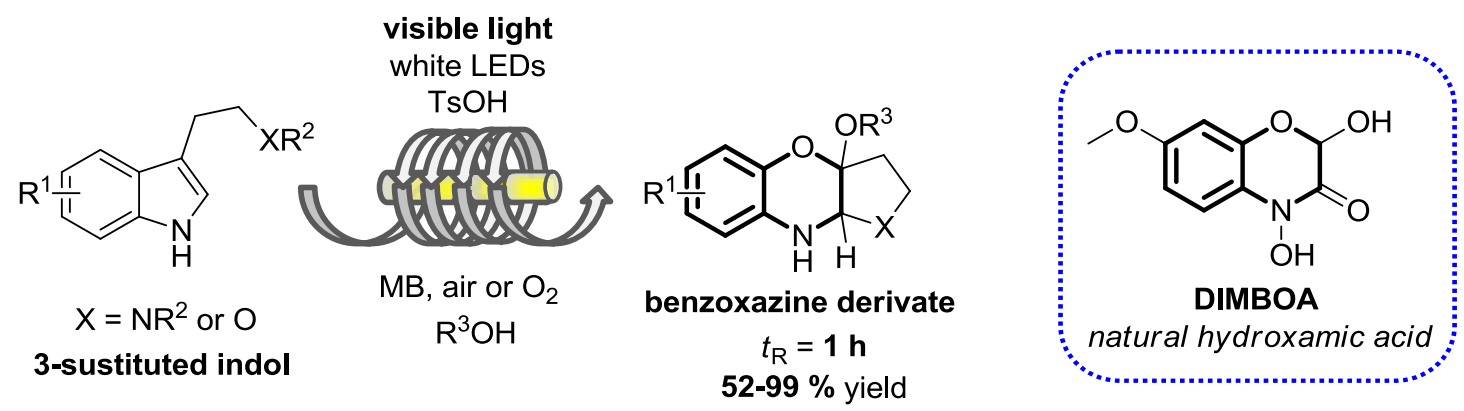

Scheme 14. Access to benzoxazines derivatives via photoredox catalysis.

Jamison and co-workers have used photoredox catalysis in flow for amino acid synthesis, ${ }^{77}$ by activation of carbon dioxide. Despite its abundance, availability and sustainability, the use of $\mathrm{CO}_{2}$ as a carbon building block is limited by its low reactivity and high stability. However, carbon-carbon bond formation could be carried out through a single-electron reduction of $\mathrm{CO}_{2}$ using para-terphenyl as photoredox catalysts. The $\mathrm{CO}_{2}$ radical anion intermediate is coupled with an $\alpha$-amino radical formed by SET and subsequent deprotonation. Additionally, the protocol involves a continuous segmented 
photo-flow setup, which is generated with a liquid solution that contains the amine substrate and the catalyst mixed with $\mathrm{CO}_{2}$ gas in line (Scheme 15).
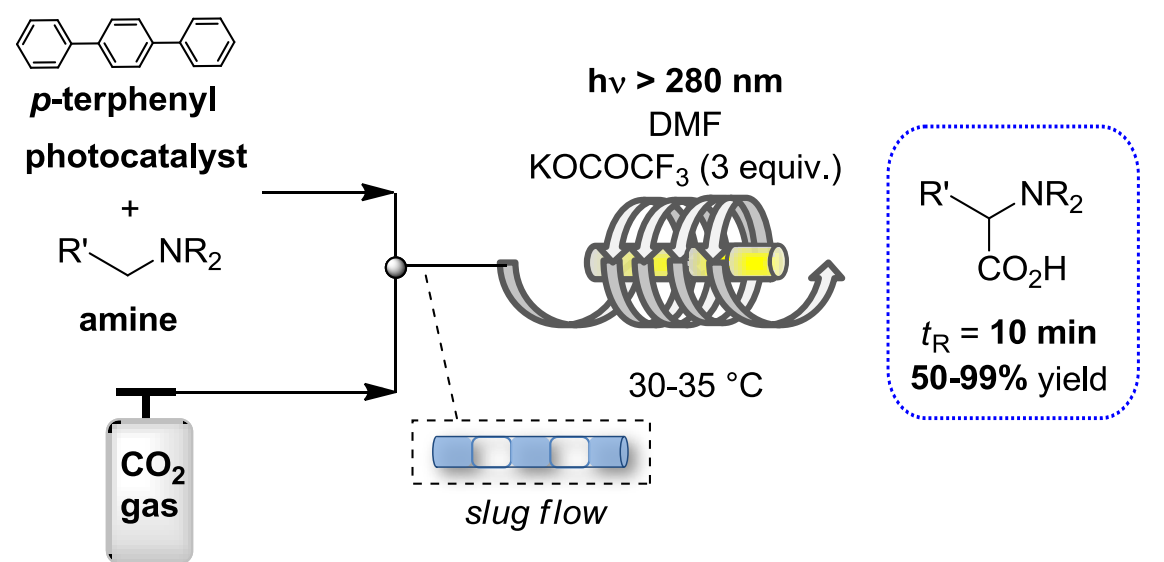

Scheme 15. Generation of $\alpha$-amino acid via photoredox catalysis using $\mathrm{CO}_{2}$ as carbon building block.

Bode et al., have reported the synthesis of morpholines, piperazines, thiomorpholines, diazepanes, and oxazepanes heterocycle derivatives, using photoredox catalysis. This protocol involves the use of commercially available SnAP reagents and aldehydes to obtain saturated N-heterocycles under continuous flow photocatalysis conditions. The methodology showed excellent functional group tolerance, allowing the scale-up of $\mathrm{N}$ heterocycles in good yield (17-57 \% yield) after 9 min (Scheme 16).

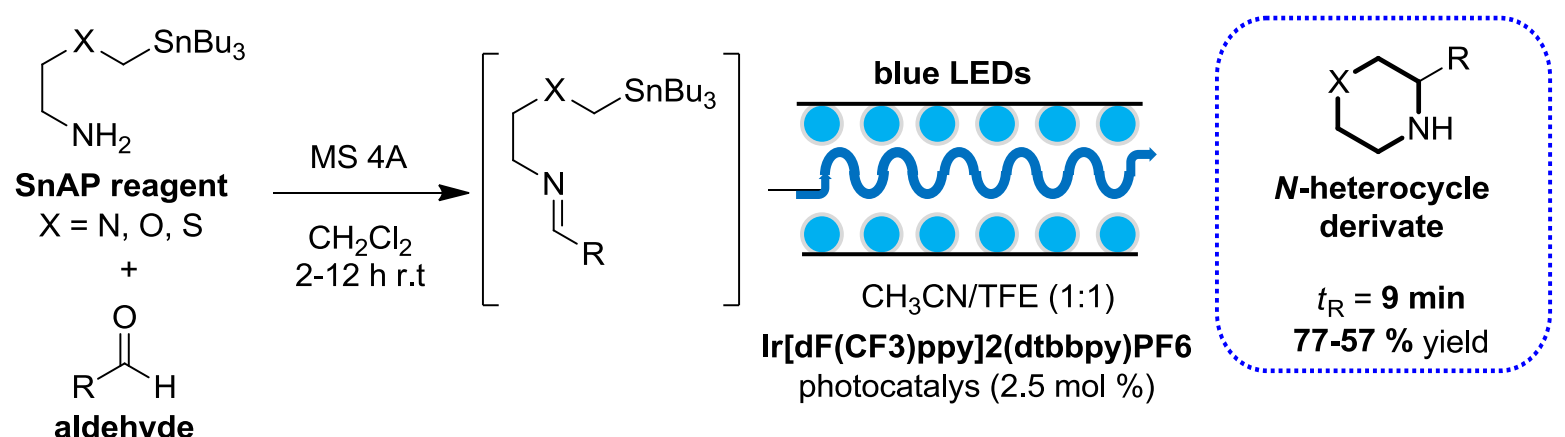

Scheme 16. Synthesis of Saturated $N$-heterocycles with SnAP reagent under photoredox catalysis in flow. 


\section{Miscellaneous Photochemical Reaction}

One of the photochemical reactions widely used in the synthesis of a valuable APIs are photo-oxygenation reactions, which have great relevance for the pharmaceutical industry. In this context, the advance in adoption of continuous processing to carry out oxidation has been significant. ${ }^{78}$ In this sense, Rossen, Poliakoff, George, and coworker, carried out a photo-oxygenation reaction to synthesize the Artemisinin antimalarial drug. ${ }^{79}$ Making use of the principles of green chemistry, the authors proposed two synthetic strategies with a continuous flow photoreactor. One of them (Method A) involves the use of liquid $\mathrm{CO}_{2}$ as solvent, toluene as co-solvent and a dualfunction solid acid/photocatalyst. Commercially available porphyrins straightforward onto the sulfonated crosslinked polystyrene ion-exchange resin Amberlyst-15 were applied, given a dual function of the Brønsted acidic and photo-catalytic needed to obtain Artemisinin via ${ }^{1} \mathrm{O}_{2}$ photo-oxygenation starting from dihydroartemisinic acid (Scheme 16). The second one (Method B) involves the use of an aqueous mixture of organic solvent, $\left[\mathrm{Ru}(\text { bpy })_{3}\right]^{2+}$ as photocatalyst and $\mathrm{H}_{2} \mathrm{SO}_{4}$ as acid in a tubular photoreactor. The mixtures result in a spontaneous crystallization of pure photoproduct Artemisinin, while the photocatalyst used plus $\mathrm{H}_{2} \mathrm{SO}_{4}$ remains in water phase and could be recycled (Scheme 17).

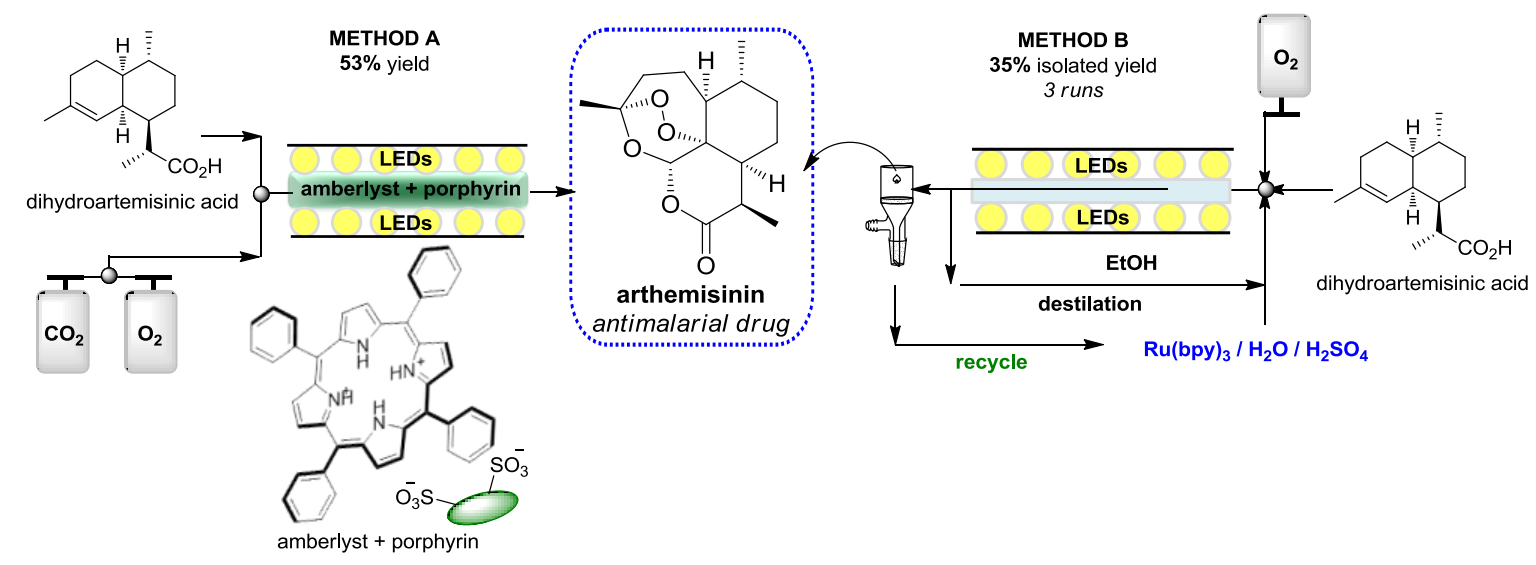


Scheme 17. Synthesis of Artemisinin carrying out photo-oxygenation reaction. Method A: Heterogeneous synthesis using liquid $\mathrm{CO}_{2}$ as solvent and a dual-function solid acid/photocatalyst. Method B: Homogeneous synthesis using recycled acid and photocatalysts.

Cochran et al. described a photochemical rearrangement of chiral oxaziridines in continuous flow to obtain chiral bicyclic lactams (Scheme 18) ${ }^{80}$ Alternatively to Schmidt and Beckmann's rearrangements, prochiral ketones can be converted to chiral oxaziridines which undergo a stereoselective photolytic rearrangement to produce the chiral lactam. Caprolactams derivatives have been reported as $\chi$-secretase inhibitors, which are involved in Alzheimer's disease ${ }^{81}$ Furthermore, their heterocyclic structure is present in compounds which show potent angiotensin-converting enzyme (ACE) inhibition activity. The authors carried out the photolysis in a continuous flow reactor, demonstrating its potential usefulness to perform this type of photochemical reaction on a larger scale.
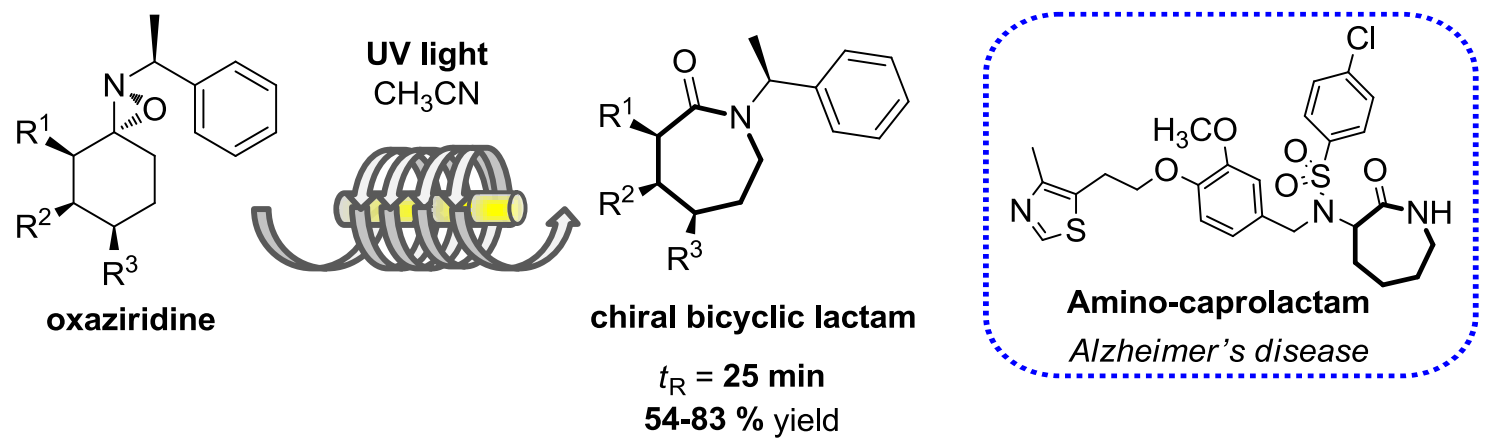

Scheme 18. Photo-rearrangement of chiral oxaziridines to obtain chiral bicyclic lactams.

Photo-rearrangements are an interesting strategy for accessing the scaffold structure in a simple way. This is the cases of photo-Favorskii rearrangement, which involves an elimination reaction coupled with a 1,2-carbon migration and subsequent nucleophilic 
attack to obtain carboxylic acid from $\alpha$-substituted ketone. In this context, Baxendale and co-worker carried out a successful synthesis of ibuprofen under continuous-flow photochemistry. ${ }^{82}$ Ibuprofen (isobutylphenyl propionic acid) is a nonsteroidal antiinflammatory drug widely used to alleviate pain, fever and inflammation. The strategy is based on the photo-Favorskii rearrangement of the appropriate halopropiophenone (achloropropiophenone) using a commercially available Vapourtec E-series flow system. Evaluating different reaction conditions such as different filters, residence times and concentration, after 20 minutes of irradiation it was possible to generate a $75 \%$ yield of ibuprofen (Scheme 19).

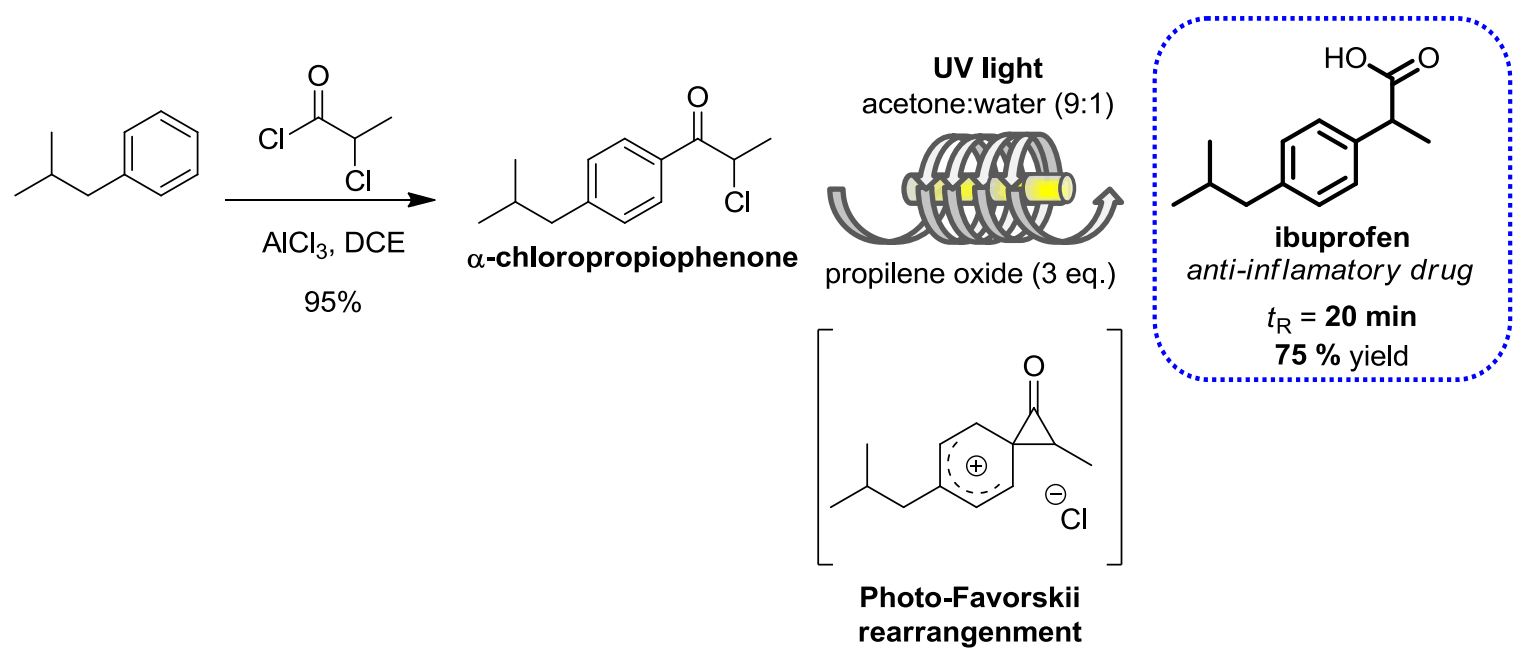

Scheme 19. Photo-Favorskii rearragenment to access ibuprofen.

Monbaliu and co-workers have reported a photochemical radical addition of isopropanol-derived to maleic or fumaric acid to obtain $\gamma$-butyrolactone scaffolds under scalable continuous-flow conditions. ${ }^{83} \gamma$-Butyrolactones are reported to be building blocks for the preparation of biologically relevant molecules, and are present in many natural products, such as paraconic acids, trisubstituted $\gamma$-butyrolactones which show antibiotic and antitumor properties. ${ }^{84}$ With a simple methodology, this kind of heterocyclic compound could be synthesized using a lab-scale mesofluidic reactor with 
inline NMR spectroscopy, enabling fast optimization of the reaction parameters. Under optimized conditions, a family of $\gamma$-butyrolactones was obtained on a scale-up methodology to $12 \mathrm{~g} \mathrm{day}^{-1}$ in a short time, with moderate to high isolated yields (47-76 \%) (Scheme 20).

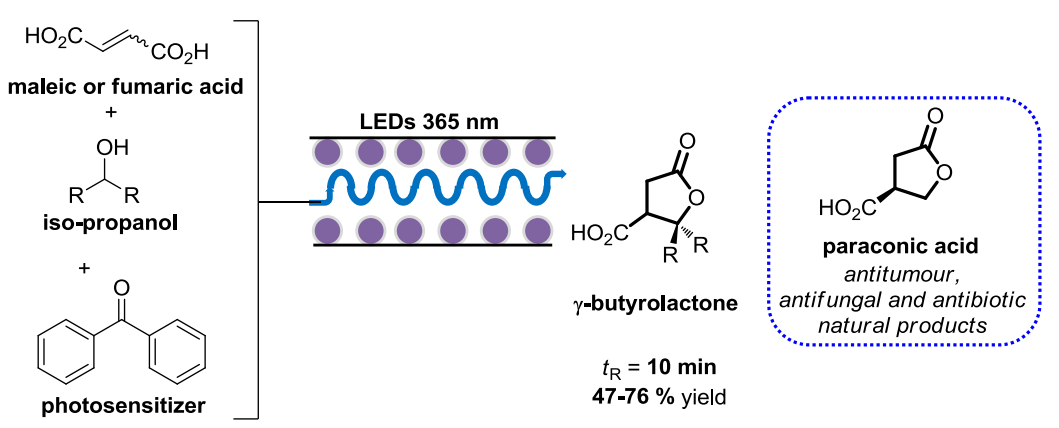

Scheme 20. Synthesis of trisubstituted $\gamma$-butyrolactones via photochemical radical addition reaction.

Interestedly, the group from Monbaliu reported inter- and intramolecular strategies for the diasteroselective preparation of threo-methylphenidate hydrochloride which involves a continuous-flow $\mathrm{Rh}$-catalyzed intermolecular $\mathrm{C}-\mathrm{H}$ carbene insertion or a thermal or photochemical intramolecular C-H carbene insertion. Specifically, in the last example, the photochemical intramolecular insertion originates $\beta$-lactam intermediate, which, after methanolysis processing under acidic conditions, forms methylphenidate hydrochloride. ${ }^{85}$ The interest of methylphenidate lies in its pharmacological properties when acting as a norepinephrine-dopamine reuptake inhibitor, and is widely uses to treat attention deficit hyperactivity disorder (ADHD) and narcolepsy. Furthermore, the different stereoisomers have different pharmacological profiles. For this reason, the development of new processes for the stereoselective preparation of methylphenidate are very important. In this way, the photochemical decomposition of tosylhydrazone is carried out in presence of 1,8-diazabicyclo[5.4.0]undec-7-ene (DBU), resulting in 97\% 
of the conversion after 60 minutes of residence time, and with a productivity of $0.3 \mathrm{~g} \mathrm{~h}^{-1}$ (Scheme 21)

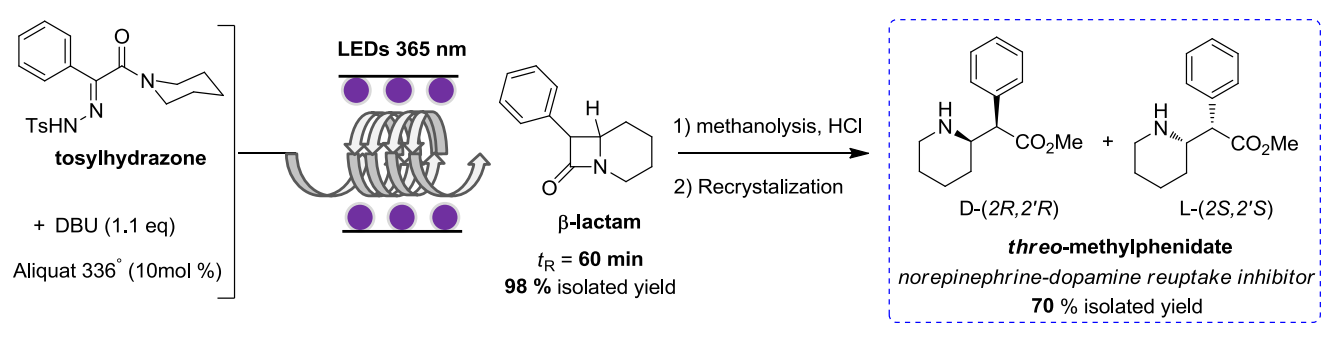

Scheme 21. Employing photochemical intramolecular insertion C-H carbene insertion to obtain methylphenidate.

\section{Multi-step Continuous-Flow Synthetic Applications involving a Photochemical Step}

The art of total synthesizing to access complex organic molecules such as natural product is defined by the concept of "ideal synthesis", which should be the focus in providing large quantities of complex products, minimizing work and materials expenses. In this way, the use of multistep flow sequences has allowed to improvement of several synthetic applications. ${ }^{86}$ Besides, numerous single-step photo-flow transformations have been reported in recent years, dealing with the generation of unstable diazo compounds for use in divergent proto-deboronative and oxidative $\mathrm{C}\left(\mathrm{sp}_{2}\right)$ $\mathrm{C}\left(\mathrm{sp}_{3}\right)$ cross-coupling processes ${ }^{87}$ such as arylation of cysteine by photoredox catalysis ${ }^{88}$ decarboxylative coupling of amino acids with aryl halides using a dual catalysis methodology (combination of photoredox and Ni catalysis) ${ }^{89}$ preparation of organosilanes through hydrosilylation by tetrabutylammonium decatungstatephotocatalyzed activation of the Si-H bond. ${ }^{90}$

In spite of this, only a few examples of synthetic sequences in flow which include at least one photochemical step were found in the literature. In this section, multistep 
continuous-flow photochemical transformations are surveyed, in order to provide a perspective, and hopefully an inspiration for future research, in the quest for the ideal synthesis.

The real challenge of multi-step continuous-flow reactions involves the ability to combine different reactions, taking into account solvent compatibility, flow-rate synergy and by-product effects. In this context, Jamison and co-worker have developed an interesting three-step-in-one-flow protocol to produce 2'-deoxy and 2',3'dideoxynucleosides (Scheme 22). ${ }^{91}$ Nucleosides and nucleoside analogs have long been an important class of medicinal agents, possessing anticancer and antiviral activity. ${ }^{92,93}$ The synthesis protocol involves a sequence of glycosylation, photo-sensitized deoxygenation and deprotection reactions. In the first step, 2'-mtrifluoromethylbenzoate ribose together with $5 \mathrm{~mol}-\%$ of pyridinium triflate salts as catalysts and a silylated nucleoside base were introduced into the T-mixer, to carry out the Vorbrüggen glycosilation. The 2-O-deoxyribonuleoside obtained was directly mixed with the 3,6-dimethoxy-9-ethylcarbazole photosensitizer to bring about the photoinduced electron transfer deoxygenation reaction. Finally, a solution of $\mathrm{NaOH}$ was introduced via a T-mixer to the exiting stream from the second step to carry out the deprotection reaction. With the continuous multi-step, one-flow sequence the thymidine and anti-HIV drug edoxudine were successfully synthesized with $72 \%$ and $66 \%$ yields respectively. 


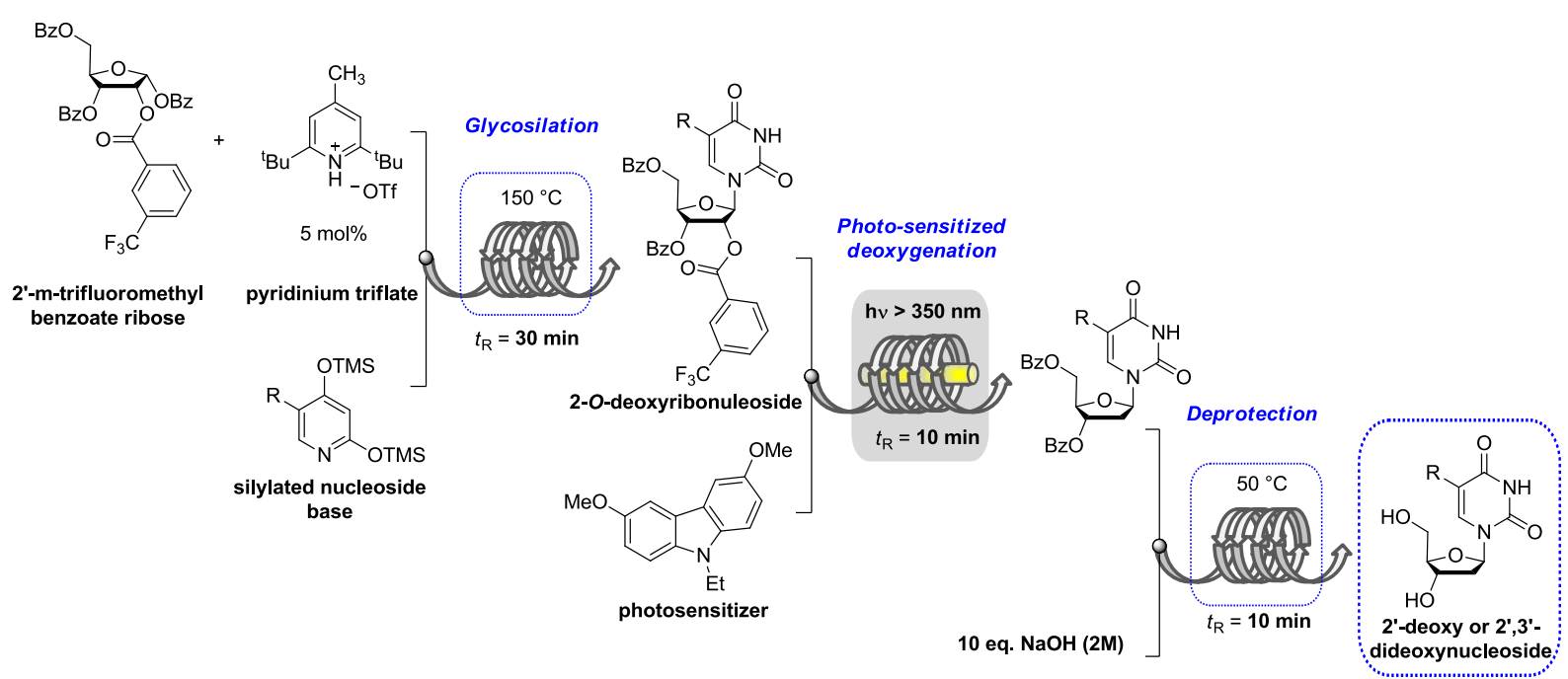

Scheme 22. Synthesis of 2'-deoxy and 2',3'-dideoxynucleosides via a sequence of glycosylation, photo-sensitized deoxygenation and deprotection reactions.

Very recently, a review has been published of photochemical Wolff rearrangements and their application in integrated microflow syntheses. ${ }^{94}$ In the photo-Wolff rearrangement, an $\alpha$-diazocarbonyl compound undergoes rearrangement, obtaining a ketene intermediate, which can react with different nucleophiles. In this way, an interesting application based on an Arndt-Eistert homologation sequence was developed by Kappe et al. for the synthesis of $\beta$-amino acids from the respective protected $\alpha$-amino acids (Scheme 23). ${ }^{95}$ The process involves a fully continuous 4 successive reaction: amino acid activation by anhydride, acylation of diazomethane, the photo-induced Wolf rearrangement, and nucleophilic addition. Without any doubt, one of the challenges of Arndt-Eistert homologation is the safe use of diazomethane $\left(\mathrm{CH}_{2} \mathrm{~N}_{2}\right)$, which is a very toxic reagent, highly irritating, a carcinogenic compound, volatile and exceedingly sensitive to heat, light, and shock, leading to explosive decomposition. For this reason, carrying out the Arndt-Eistert homologation in the continuous-flow sequence has allowed solution of the problems associated with the handling of a dangerous reagent and with photochemistry, such as scalability and over-irradiation. Therefore, for the 
activation of $\alpha$-amino acid, a solution of the corresponding amino acid and base $\left(\mathrm{Bu}_{3} \mathrm{~N}\right)$ were mixed in a T-shaped mixer with an anhydride chloride to generate anhydride derivatives. Later, the anhydride was combined with $\mathrm{CH}_{2} \mathrm{~N}_{2}$ which was previously formed by treating Diazald (commercially available) with $\mathrm{KOH}$, to finally generate the $\alpha$-diazoketone. The remaining diazomethane was removed by introducing the reaction solutions into a gas-permeable tube (AF 2400) which was immersed in acetic acid. Before performing the photo-induced Wolf rearrangement, the corresponding diazoketone was mixed with a solution of $\mathrm{H}_{2} \mathrm{O} / \mathrm{THF}$, and then sent through the photoreactor, obtaining the corresponding $\beta$-amino acid.

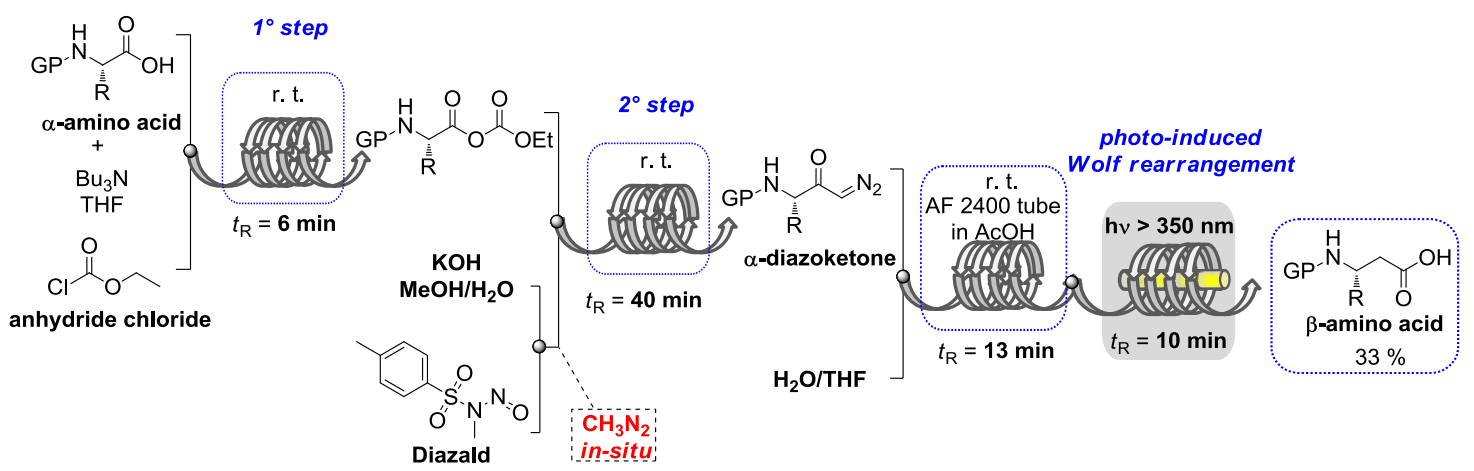

Scheme 23. Preparation of $\beta$-amino acids through four successive reactions: amino acid activation by anhydride, acylation of diazomethane, photo-induced Wolf rearrangement, and nucleophilic addition.

Beeler et al. developed an efficient photo-deprotection group of amine, and afterwards they applied it in a multistep flow synthesis. ${ }^{96}$ Then 2-methoxy-hydroxymethylxanthene was used as protecting group and chromophore, showing an overall stability under a variety of conditions and compatibility with organic solvent. Particularly, a piperazinylcarbonyl-piperidine derivative was synthesized by a three-step-in-one-flow protocol, which includes amidation, photo-deprotection and acylation reactions. In the first step an activated ester and diisopropylethylamine (DIPEA) were combined with a 
protected piperazine at a $\mathrm{T}$-junction, and subsequently the reaction mixture was made to flow into the photochemical reactor to carry out the photo-deprotection. Finally, a solution of benzoyl chloride in dicloroethane was introduced to exiting stream from the second step to conduct the acylation reaction, obtaining the piperazinylcarbonylpiperidine derivative in a $42 \%$ yield with an overall residence time of 55 min (Scheme 24).

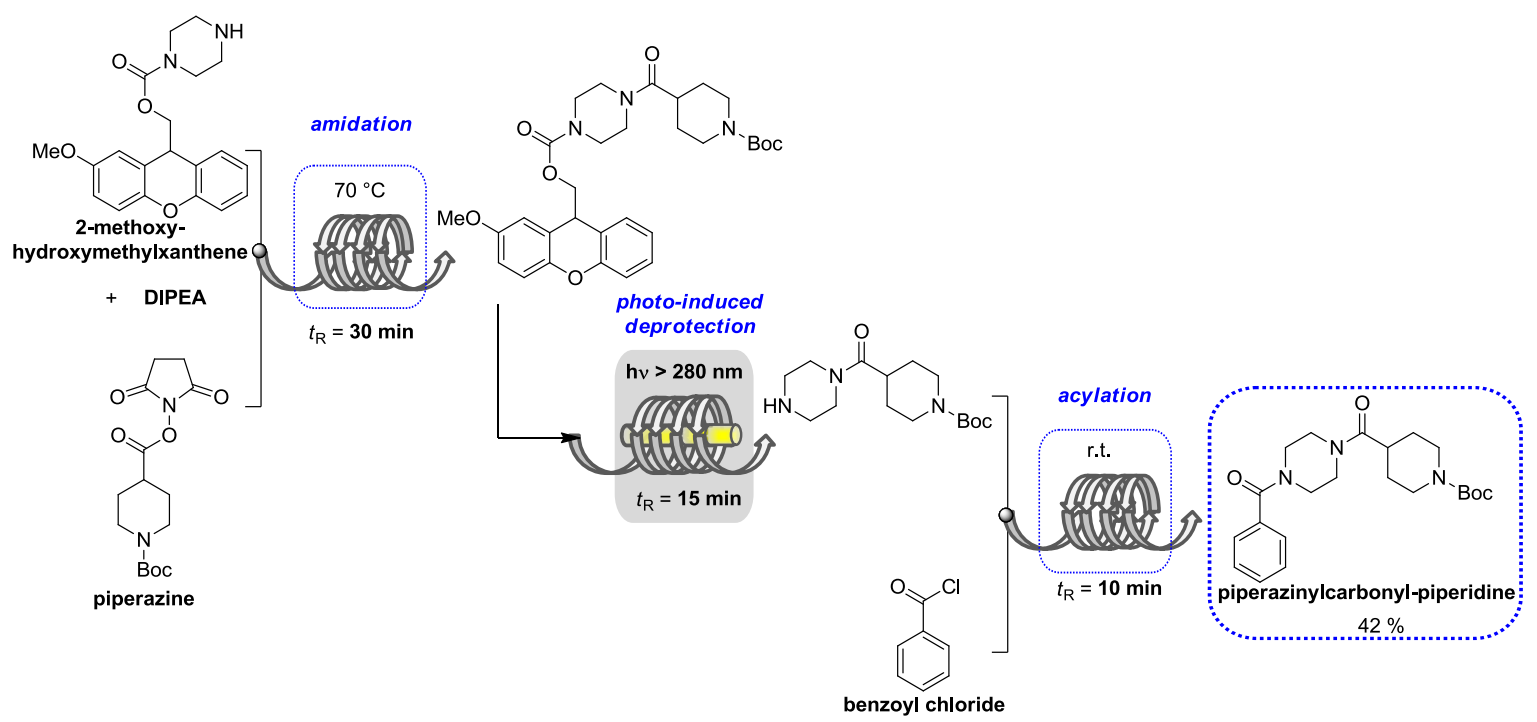

Scheme 24. Continuous-flow multistep synthesis of piperazinylcarbonyl-piperidine via amidation, photo-deprotection and acylation reactions.

Once again, the synthesis of the natural product artemisinin plays an important role in the development of different methods and technologies for industrial-scale production. ${ }^{97}$ Taking into account that about $1 \%$ of artemisinin is extracted from the Artemisia annua plant, numerous efforts have been made to implement processes that mimic the biosynthetic pathway. For this reason, considering that reagents and catalysts can be found in natural sources, the plant waste material has also been used to prepare artemisinin or another artemisinin-derived APIs, and to carry the desired transformation out directly. With these in mind, Seeberger, Gilmore et al. have developed a green chemical protocol to synthesize artemisinin which involves the use of 
dihydroartemisinic acid (DHAA) as well as chlorophyll (as photosensitizer) contained in the plant extract. ${ }^{98}$ In a two-step-in-one-flow protocol, the crude extract containing DHAA in presence of trifluoroacetic acid was combined with oxygen in a T-shaped mixer and the photoreaction was conducted under visible light irradiation at $-20{ }^{\circ} \mathrm{C}$. The second reaction line was kept at room temperature and after 10 min of residence time, the corresponding artemisinin is generated (Scheme 25).
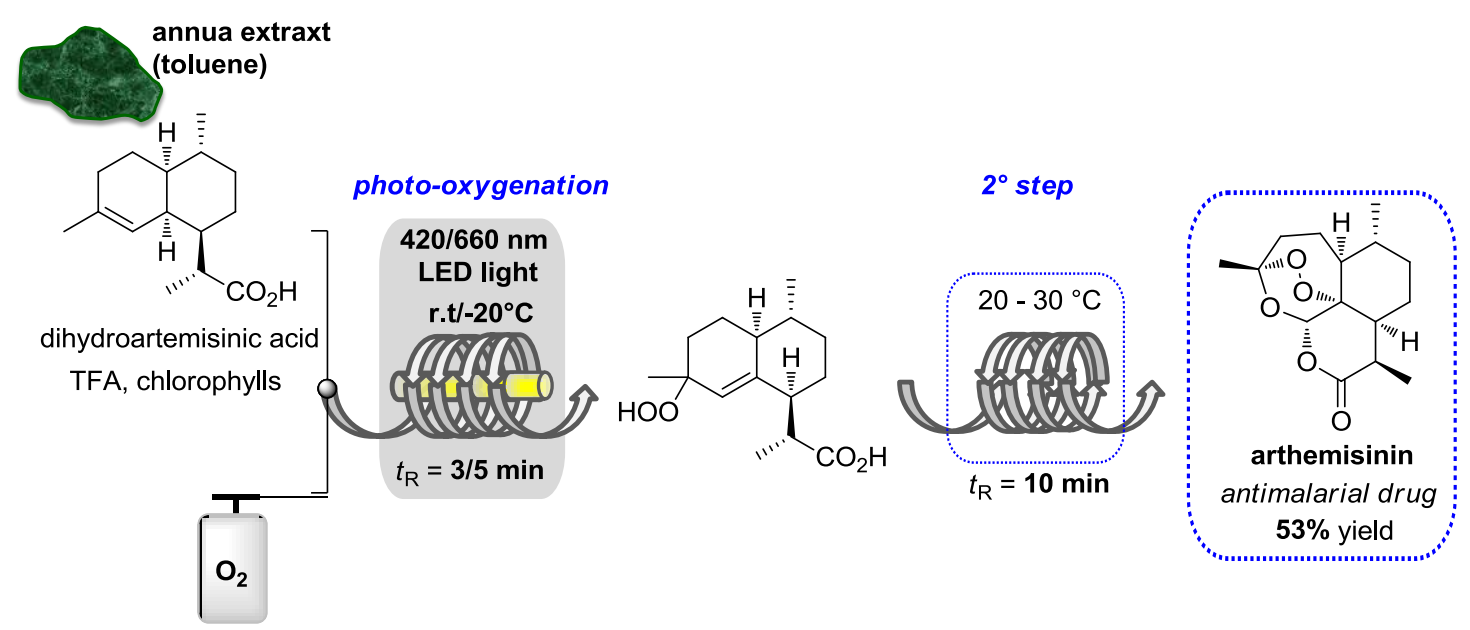

Scheme 25. Two-step-in-one-flow protocol to prepare Arthemisinin antimalarial drug.

The oxidant singlet oxygen is a key reactive intermediate involved in the photooxidation of natural products. Particularly, primary or secondary amines can be photooxidized by ${ }^{1} \mathrm{O}_{2}$ to generate imine intermediate, and if this imine is trapped by cyanide anion, $\alpha$-amino-nitrile is obtained as final product. Using this synthetic strategy, Gilmore et al. built hydantoins heterocycles with a two-step continuous-flow sequence of photo-oxidation and carboxylation rearrangement. ${ }^{99}$ Hydantoin derivatives possess important biochemical and pharmacological properties such as anti-inflammatory, antiHIV, anticonvulsant, antihypertensive and are used to treat many human diseases. Taking into account the relevance of this heterocycle, its synthetic approach in continuous-flow chemistry suggests an alternative framed within the principles of green 
chemistry, carrying out biphasic gas/liquid reactions with an $\mathrm{O}_{2}$ and $\mathrm{CO}_{2}$ source and with a highly atom economy, using light as a traceless reagent and an eco-friendly solvent. After of a residence time of 24 minutes, different benzylic and aliphatic unprotected hydantoins were obtained in good yields (Scheme 26).

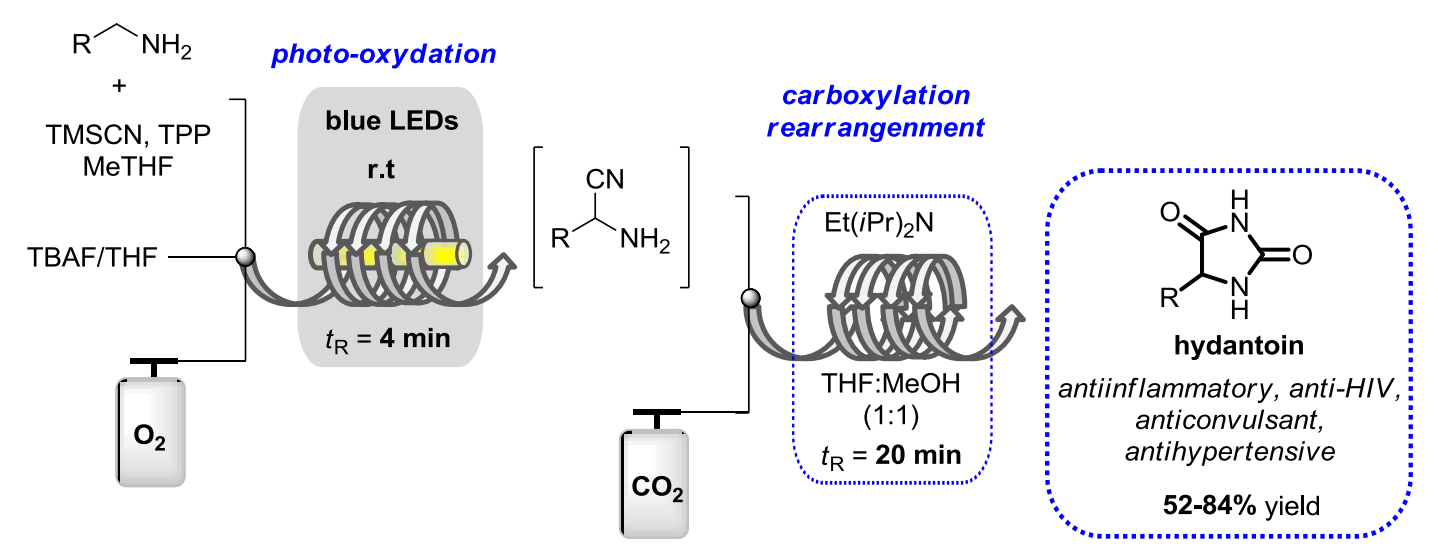

Scheme 26. Two-step continuous-flow sequence to access hydantoins derivatives.

In the previous section we explained a protocol developed by Collins and co-workers to synthesize a family of carzabazole heterocycles (Scheme 12). Currently, the same group is extending its strategy to access a more complex carbazole structure using two-step continuous-flow processes. ${ }^{100}$ Via photochemical decomposition of azides, a variety of carbazoles can be obtained, which are involved in a subsequent transformation to generate a family of the alkaloid clausine $\mathrm{C}$, with interesting biological properties such as antimalarial, antibacterial and anti-HIV. On the one hand, a bromide-substituted azide is photo-decomposed under continuous flow process using purple LEDs. Subsequent Suzuki cross-coupling with heterogeneous palladium-based catalyst (SiliaCat) and boronic acids was carried out, obtaining a family of aryl and heteroaryl carabazoles with good yield (36-74\%) after approximately 4h (Scheme 27, Protocol 1). On the other hand, exploiting the bromo functionality of carabazole derivatives, Collins et al. carried out a second two-step protocol, attaching a subsequent dual-photocatalyst 
reaction. For this purpose a Ni-catalysis cross-coupling with bromo-carbazole, potassium alkyl trifluoroborates and organic dye 4CzIPN as photoredox-catalyst were performed. Under blue LEDs irradiation, after $3 \mathrm{~h}$ approximately, a new carbazole derivative was generated (Scheme 27, Protocol 2).

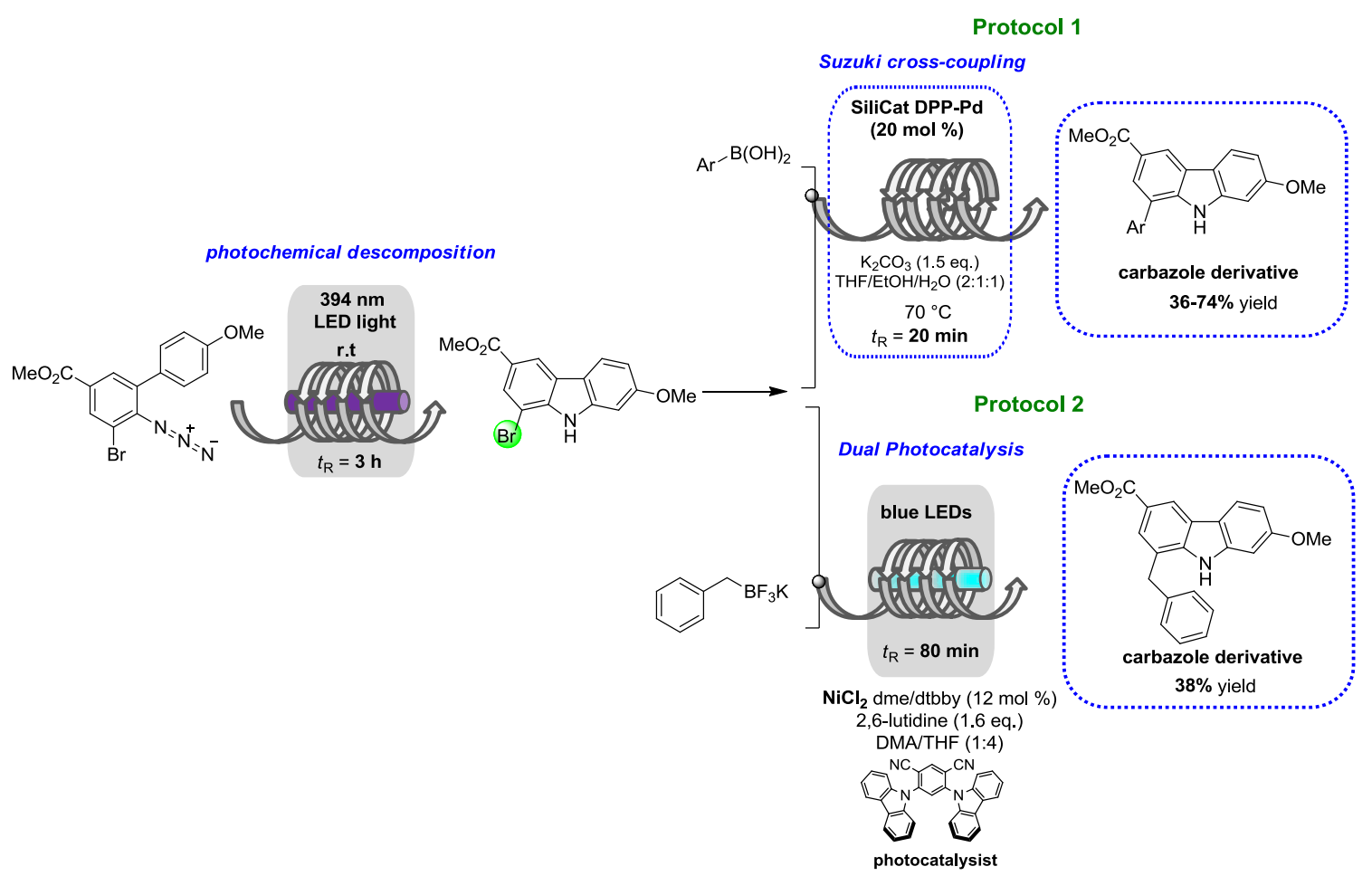

Scheme 27. Two-step protocols in continuous flow. Protocol 1: with a Suzuki crosscoupling step. Protocol 2: with a Nickel/photoredox cross-coupling.

\section{Outlook}

It is clear that photochemistry has earned an important place in organic synthesis, showing its versatility to access complex molecular structures in a simple way. Furthermore, considering that its applications have been found to be framed within the green chemistry and engineering principles, activation with light of different organic compounds has emerged as an attractive synthetic tool. In this context, flow chemistry has acted as a bridge between the past and the future of organic synthesis. The challenge to photochemists and engineers was the development of methodologies or processes 
relevant for the pharmaceutical industry. The use of flow photochemistry has allowed the overcoming of some setbacks, leading to the acquisition of APIs in milder, safer and more sustainable conditions. Additionally, with the technological advances, photochemical reactions have demonstrated that they can successfully be introduced in multi-step processes. Today it only remains for us to calculate the best route to drive total synthesis. For this reason, future challenges will be related to the combination, in individual modules, of multi-catalysis methodologies (photocatalysis, enzymatic catalysis, organocatalysis and metal catalysis) under continuous flow conditions, to access chiral compounds with biological interest. Using nature as a source of inspiration and applying flow chemistry, scientists should only look at the light on the horizon, order their steps, and keep walking.

\section{Acknowledgements}

This work was supported in part by Consejo Nacional de Investigaciones Científicas y Técnicas (CONICET), Secretaría de Ciencia y Tecnología (SeCyT), Universidad Nacional de Córdoba (UNC) and Fondo para la Investigación Científica y Tecnológica Argentina (FONCyT). FP gratefully acknowledges receipt of a fellowship from CONICET.

\section{References}

(1) Kärkäs, M. D.; Porco, J. A.; Stephenson, C. R. J. Photochemical Approaches to Complex Chemotypes: Applications in Natural Product Synthesis. Chem. Rev. 2016, 116, 9683-9747.

(2) Handbook of Synthetic Photochemistry; Albini, A., Fagnoni, M., Eds.; WileyVCH Verlag GmbH \& Co. KGaA: Weinheim, Germany, 2009. 
(3) Romero, N. A.; Nicewicz, D. A. Organic Photoredox Catalysis. Chem. Rev. 2016, $116,10075-10166$.

(4) New Methodologies and Techniques for a Sustainable Organic Chemistry; Alessandro Mordini and Ferenc Faigl, Ed.; Springer: Siena, Italy, 2005.

(5) Noël, T. Photochemical Processes in Continuous-Flow Reactors; World Scientific (Europe), 2017.

(6) Cambié, D.; Bottecchia, C.; Straathof, N. J. W.; Hessel, V.; Noël, T. Applications of Continuous-Flow Photochemistry in Organic Synthesis, Material Science, and Water Treatment. Chem. Rev. 2016, 116, 10276-10341.

(7) Otake, Y.; Nakamura, H.; Fuse, S. Recent Advances in the Integrated MicroFlow Synthesis Containing Photochemical Reactions. Tetrahedron Lett. 2018, 59, 1691-1697.

(8) Noël, T. A Personal Perspective on the Future of Flow Photochemistry. J. Flow Chem. 2017, 7, 87-93.

(9) Morse, P. D.; Beingessner, R. L.; Jamison, T. F. Enhanced Reaction Efficiency in Continuous Flow. Isr. J. Chem. 2017, 57, 218-227.

(10) Lummiss, J. A. M.; Morse, P. D.; Beingessner, R. L.; Jamison, T. F. Towards More Efficient, Greener Syntheses through Flow Chemistry. Chem. Rec. 2017, $17,667-680$.

(11) Su, Y.; Kuijpers, K.; Hessel, V.; Noël, T. A Convenient Numbering-up Strategy for the Scale-up of Gas-liquid Photoredox Catalysis in Flow. React. Chem. Eng. 2016, $1,73-81$. 
(12) Kuijpers, K. P. L.; van Dijk, M. A. H.; Rumeur, Q. G.; Hessel, V.; Su, Y.; Noël, T. A Sensitivity Analysis of a Numbered-up Photomicroreactor System. React. Chem. Eng. 2017, 2, 109-115.

(13) Zhao, F.; Cambié, D.; Janse, J.; Wieland, E. W.; Kuijpers, K. P. L.; Hessel, V.; Debije, M. G.; Noël, T. Scale-up of a Luminescent Solar Concentrator-Based Photomicroreactor via Numbering-Up. ACS Sustain. Chem. Eng. 2018, 6, 422429.

(14) Loubière, K.; Oelgemöller, M.; Aillet, T.; Dechy-cabaret, O.; Prat, L. Chemical Engineering and Processing: Process Intensification Continuous-Flow Photochemistry: A Need for Chemical Engineering. Chem. Eng. Process. Process Intensif. 2016, 104, 120-132.

(15) Nauth, A. M.; Lipp, A.; Lipp, B.; Opatz, T. Sunflow: Sunlight Drives Fast and Green Photochemical Flow Reactions in Simple Microcapillary Reactors Application to Photoredox and H-Atom-Transfer Chemistry. European J. Org. Chem. 2017, 2017, 2099-2103.

(16) Nakano, M.; Nishiyama, Y.; Tanimoto, H.; Morimoto, T.; Kakiuchi, K. Remarkable Improvement of Organic Photoreaction Efficiency in the Flow Microreactor by the Slug Flow Condition Using Water. Org. Process Res. Dev. 2016, 20, 1626-1632.

(17) Josland, S.; Mumtaz, S.; Oelgemöller, M. Photodecarboxylations in an Advanced Meso-Scale Continuous-Flow Photoreactor. Chem. Eng. Technol. 2016, 39, 8187.

(18) Elgue, S.; Aillet, T.; Loubiere, K.; Conté, A.; Dechy-Cabaret, O.; Prat, L.; Horn, 
C. R.; Lobet, O.; Vallon, S. Flow Photochemistry: A Meso-Scale Reactor for Industrial Applications. Chem. Today 2015, 33, 58-61.

(19) Oelgemöller, M. Highlights of Photochemical Reactions in Microflow Reactors. Chem. Eng. Technol. 2012, 35, 1144-1152.

(20) Hook, B. D. A.; Dohle, W.; Hirst, P. R.; Pickworth, M.; Berry, M. B.; BookerMilburn, K. I. A Practical Flow Reactor for Continuous Organic Photochemistry. J. Org. Chem. 2005, 70, 7558-7564.

(21) Straathof, N. J. W.; Su, Y.; Hessel, V.; Noël, T. Accelerated Gas-Liquid Visible Light Photoredox Catalysis with Continuous-Flow Photochemical Microreactors. Nat. Protoc. 2016, 11, 10-21.

(22) Ioannou, G. I.; Montagnon, T.; Kalaitzakis, D.; Pergantis, S. A.; Vassilikogiannakis, G. A Novel Nebulizer-Based Continuous Flow Reactor: Introducing the Use of Pneumatically Generated Aerosols for Highly Productive Photooxidations. ChemPhotoChem 2017, 1, 173-177.

(23) Elliott, L. D.; Berry, M.; Harji, B.; Klauber, D.; Leonard, J.; Booker-Milburn, K. I. A Small-Footprint, High-Capacity Flow Reactor for UV Photochemical Synthesis on the Kilogram Scale. Org. Process Res. Dev. 2016, 20, 1806-1811.

(24) Britton, A. J.; Stubbs, K. A.; Weiss, G. A.; Raston, L.; Britton, J.; Stubbs, K. A.; Weiss, G. A.; Raston, C. L. Vortex Fluidic Chemical Transformations. Chem. Eur. J. 2017, 23, 13270.

(25) Gandy, M. N.; Raston, C. L.; Stubbs, K. A. Photoredox Catalysis under Shear Using Thin Film Vortex Microfluidics. Chem. Commun. 2015, 51, 11041-11044. 
(26) Clark, C. A.; Lee, D. S.; Pickering, S. J.; Poliakoff, M.; George, M. W. A Simple and Versatile Reactor for Photochemistry. Org. Process Res. Dev. 2016, 20, 1792-1798.

(27) Ciamician, G. The Photochemistry of the Future. Science 1912, 36, 385-394.

(28) Cambié, D.; Zhao, F.; Hessel, V.; Debije, M. G.; Noël, T. A Leaf-Inspired Luminescent Solar Concentrator for Energy-Efficient Continuous-Flow Photochemistry. Angew. Chemie Int. Ed. 2017, 56, 1050-1054.

(29) Zhao, F.; Cambié, D.; Hessel, V.; Debije, M. G.; Noël, T. Real-Time Reaction Control for Solar Production of Chemicals under Fluctuating Irradiance. Green Chem. 2018, 2459-2464.

(30) Kuijpers, K.; Bottecchia, C.; Cambie, D.; Drummen, K.; Koenig, N.; Noel, T. A Fully Automated Continuous-Flow Platform for Fluorescence Quenching Studies and Stern-Volmer Analysis. Angew. Chemie Int. Ed. 2018, DOI: 10.1002/anie.201805632.

(31) Coley, C. W.; Abolhasani, M.; Lin, H.; Jensen, K. F. Material-Efficient Microfluidic Platform for Exploratory Studies of Visible-Light Photoredox Catalysis. Angew. Chemie - Int. Ed. 2017, 56, 9847-9850.

(32) Hsieh, H.-W.; Coley, C. W.; Baumgartner, L. M.; Jensen, K. F.; Robinson, R. I. Photoredox Iridium-Nickel Dual-Catalyzed Decarboxylative Arylation CrossCoupling: From Batch to Continuous Flow via Self-Optimizing Segmented Flow Reactor. Org. Process Res. Dev. 2018, 22, 542-550.

(33) Pieber, B.; Kappe, C. O. Aerobic Oxidations in Continuous Flow. In Top 
Organomet Chem; 2015; Vol. 48, pp 97-136.

(34) Gemoets, H. P. L.; Su, Y.; Shang, M.; Hessel, V.; Luque, R.; Noël, T. Liquid Phase Oxidation Chemistry in Continuous-Flow Microreactors. Chem. Soc. Rev. 2016, $45,83-117$.

(35) Synthetic Organic Photochemistry; Griesbeck, Axel G. and Mattay, J., Ed.; 2005; Vol. 33.

(36) Vassilikogiannakis, G. Furans and Singlet Oxygen - Why There Is More to Come from This Powerful Partnership. Chem. Commun. 2014, 50, 15480-15498.

(37) Loponov, K. N.; Lopes, J.; Barlog, M.; Astrova, E. V.; Malkov, A. V.; Lapkin, A. A. Optimization of a Scalable Photochemical Reactor for Reactions with Singlet Oxygen. Org. Process Res. Dev. 2014, 18, 1443-1454.

(38) Mendoza, C.; Emmanuel, N.; Páez, C. A.; Dreesen, L.; Monbaliu, J. C. M.; Heinrichs, B. Transitioning from Conventional Batch to Microfluidic Processes for the Efficient Singlet Oxygen Photooxygenation of Methionine. J. Photochem. Photobiol. A Chem. 2018, 356, 193-200.

(39) Emmanuel, N.; Mendoza, C.; Winter, M.; Horn, C. R.; Vizza, A.; Dreesen, L.; Heinrichs, B.; Monbaliu, J. C. M. Scalable Photocatalytic Oxidation of Methionine under Continuous-Flow Conditions. Org. Process Res. Dev. 2017, $21,1435-1438$.

(40) Shvydkiv, O.; Jähnisch, K.; Steinfeldt, N.; Yavorskyy, A.; Oelgemöller, M. Visible-Light Photooxygenation of $\alpha$-Terpinene in a Falling Film Microreactor. Catal. Today 2018, 308, 102-118. 
(41) Darren S. Lee, Zacharias Amara, Charlotte A. Clark, Zeyuan Xu, Bruce Kakimpa, H. P. M.; George, S. J. P. M. P. and M. W. Continuous PhotoOxidation in a Vortex Reactor: Efficient Operations Using Air Drawn from the Laboratory. Org. Process Res. Dev. 2017, 21, 1042-1050.

(42) Laudadio, G.; Govaerts, S.; Wang, Y.; Ravelli, D.; Koolman, H. F.; Fagnoni, M.; Djuric, S. W.; Noël, T. Selective C(sp3)-H Aerobic Oxidation Enabled by Decatungstate Photocatalysis in Flow. Angew. Chemie - Int. Ed. 2018, 57, 40784082.

(43) Porta, R.; Benaglia, M.; Puglisi, A. Flow Chemistry: Recent Developments in the Synthesis of Pharmaceutical Products. Org. Process Res. Dev. 2016, 20 (1), 2-25.

(44) Gutmann, B.; Cantillo, D.; Kappe, C. O. Continuous-Flow Technology-A Tool for the Safe Manufacturing of Active Pharmaceutical Ingredients. Angew. Chemie Int. Ed. 2015, 54, 6688-6728.

(45) Poplata, S.; Tröster, A.; Zou, Y. Q.; Bach, T. Recent Advances in the Synthesis of Cyclobutanes by Olefin [2+2] Photocycloaddition Reactions. Chemical Reviews. 2016, 9748-9815.

(46) Reem Telmesani, Sung H. Park, Tessa Lynch-Colameta, and A. B. B. [2+2] Photocycloaddition of Cinnamates in Flow and Development of a Thiourea Catalyst. Angew. Chem. Int. Ed. 2015, 54, 11521-11525.

(47) Steri, R.; Rupp, M.; Proschak, E.; Schroeter, T.; Zettl, H.; Hansen, K.; Schwarz, O.; Müller-Kuhrt, L.; Müller, K. R.; Schneider, G.; et al. Truxillic Acid Derivatives Act as Peroxisome Proliferator-Activated Receptor $\gamma$ Activators. Bioorganic Med. Chem. Lett. 2010, 20, 2920-2923. 
(48) Ma, G. L.; Xiong, J.; Yang, G. X.; Pan, L. L.; Hu, C. L.; Wang, W.; Fan, H.; Zhao, Q. H.; Zhang, H. Y.; Hu, J. F. Biginkgosides A-I, Unexpected Minor Dimeric Flavonol Diglycosidic Truxinate and Truxillate Esters from Ginkgo Biloba Leaves and Their Antineuroinflammatory and Neuroprotective Activities. J. Nat. Prod. 2016, 79, 1354-1364.

(49) Wang, M. L.; Yu, G.; Yi, S. P.; Zhang, F. Y.; Wang, Z. T.; Huang, B.; Su, R. Bin; Jia, Y. X.; Gong, Z. H. Antinociceptive Effects of Incarvillateine, a Monoterpene Alkaloid from Incarvillea Sinensis, and Possible Involvement of the Adenosine System. Sci. Rep. 2015, 5, 1-11.

(50) Serrano, E.; Juan, A.; García-Montero, A.; Soler, T.; Jiménez-Márquez, F.; Cativiela, C.; Gomez, M. V.; Urriolabeitia, E. P. Stereoselective Synthesis of 1,3Diaminotruxillic Acid Derivatives: An Advantageous Combination of C-HOrtho-Palladation and On-Flow [2+2]-Photocycloaddition in Microreactors. Chem. - A Eur. J. 2016, 22, 144-152.

(51) Blanco-Ania, D.; Gawade, S. A.; Zwinkels, L. J. L.; Maartense, L.; Bolster, M. G.; Benningshof, J. C. J.; Rutjes, F. P. J. T. Rapid and Scalable Access into Strained Scaffolds through Continuous Flow Photochemistry. Org. Process Res. Dev. 2016, 20, 409-413.

(52) Blackham, E. E.; Booker-Milburn, K. I. A Short Synthesis of ( \pm )-3Demethoxyerythratidinone by Ligand-Controlled Selective Heck Cyclization of Equilibrating Enamines. Angew. Chemie Int. Ed. 2017, 56, 6613-6616.

(53) Merlugo, L.; Santos, M. C.; Sant'Anna, L. S.; Cordeiro, E. W. F.; Batista, L. A. C.; Miotto, S. T. S.; Garcia, C. V.; Moreira, C. M.; Mendez, A. S. L. Alkaloids in 
Erythrina by UPLC-ESI-MS and In Vivo Hypotensive Potential of Extractive Preparations. Evidence-Based Complement. Altern. Med. 2015, 2015, 1-12.

(54) Popsavin, V.; Srećo, B.; Benedeković, G.; Francuz, J.; Popsavin, M.; Kojić, V.; Bogdanović, G. Design, Synthesis and Antiproliferative Activity of Styryl Lactones Related to (+)-Goniofufurone. Eur. J. Med. Chem. 2010, 45, 28762883.

(55) Ralph, M.; Ng, S.; Booker-Milburn, K. I. Short Flow-Photochemistry Enabled Synthesis of the Cytotoxic Lactone (+)-Goniofufurone. Org. Lett. 2016, 18, 968971.

(56) Yueh, H.; Gao, Q.; Porco, J. A.; Beeler, A. B. A Photochemical Flow Reactor for Large Scale Syntheses of Aglain and Rocaglate Natural Product Analogues. Bioorganic Med. Chem. 2017, 25, 6197-6202.

(57) An, F. L.; Wang, X. B.; Wang, H.; Li, Z. R.; Yang, M. H.; Luo, J.; Kong, L. Y. Cytotoxic Rocaglate Derivatives from Leaves of Aglaia Perviridis. Sci. Rep. 2016, 6, 6-17.

(58) Fang, Y.; Tranmer, G. K. Continuous Flow Photochemistry as an Enabling Synthetic Technology: Synthesis of Substituted-6(5H)-Phenanthridinones for Use as poly(ADP-Ribose) Polymerase Inhibitors. Med. Chem. Comm 2016, 7, 720724.

(59) Koolman, H. F.; Braje, W. M.; Haupt, A. Synthesis of 4,4'-Disubstituted and Spiro-Tetrahydroquinolines via Photochemical Cyclization of Acrylanilides and the First Synthesis of ( \pm - Trans -Vabicaserin. Synlett 2016, 27, 2561-2566. 
(60) Liu, J.; Ogden, A.; Comery, T. A.; Spiros, A.; Roberts, P.; Geerts, H. Prediction of Efficacy of Vabicaserin, a 5-HT2C Agonist, for the Treatment of Schizophrenia Using a Quantitative Systems Pharmacology Model. CPT Pharmacometrics Syst. Pharmacol. 2014, 3, e111.

(61) Scott, J. D.; Williams, R. M. Chemistry and Biology of the Tetrahydroisoquinoline Antitumor Antibiotics. Chem. Rev. 2002, 102, 16691730.

(62) Lisiecki, K.; Czarnocki, Z. Flow Photochemistry as a Tool for the Total Synthesis of (+)-Epigalcatin. Org. Lett. 2018, 20, 605-607.

(63) Castro, M. A.; García, P. A.; Hernández, Á. P.; Díez, D. An Overview on Heterocyclic Podophyllotoxin Derivatives. Targets Heterocycl. Syst. 2015, 19, 28-61.

(64) Ashley, W. L.; Timpy, E. L.; Coombs, T. C. Flow Photo-Nazarov Reactions of 2Furyl Vinyl Ketones: Cyclizing a Class of Traditionally Unreactive Heteroaromatic Enones. J. Org. Chem. 2018, 83, 2516-2529.

(65) Haimowitz, T.; Fitzgerald, M. E.; Winkler, J. D. Studies Directed toward the Synthesis of Nakadomarin A. Tetrahedron Lett. 2011, 52, 2162-2164.

(66) Staveness, D.; Bosque, I.; Stephenson, C. R. J. Free Radical Chemistry Enabled by Visible Light-Induced Electron Transfer. Acc. Chem. Res. 2016, 49, 22952306.

(67) Nicholls, T. P.; Leonori, D.; Bissember, A. C. Applications of Visible Light Photoredox Catalysis to the Synthesis of Natural Products and Related 
Compounds. Nat. Prod. Rep. 2016, 33, 1248-1254.

(68) Douglas, J. J.; Sevrin, M. J.; Stephenson, C. R. J. Visible Light Photocatalysis: Applications and New Disconnections in the Synthesis of Pharmaceutical Agents. Org. Process Res. Dev. 2016, 20, 1134-1147.

(69) Caron, A.; Hernandez-Perez, A. C.; Collins, S. K. Synthesis of a Carprofen Analogue Using a Continuous Flow UV-Reactor. Org. Process Res. Dev. 2014, $18,1571-1574$.

(70) Bashir, M.; Bano, A.; Ijaz, A. S.; Chaudhary, B. A. Recent Developments and Biological Activities of N-Substituted Carbazole Derivatives: A Review. Molecules 2015, 20, 13496-13517.

(71) Hernandez-Perez, A. C.; Caron, A.; Collins, S. K. Photochemical Synthesis of Complex Carbazoles: Evaluation of Electronic Effects in Both UV- and VisibleLight Methods in Continuous Flow. Chem. - A Eur. J. 2015, 21, 16673-16678.

(72) Parisien-Collette, S.; Cruché, C.; Abel-Snape, X.; Collins, S. K. Photochemical Intramolecular Amination for the Synthesis of Heterocycles. Green Chem. 2017, $19,4798-4803$.

(73) Swift, E. C.; Williams, T. M.; Stephenson, C. R. J. Intermolecular Photocatalytic C-H Functionalization of Electron-Rich Heterocycles with Tertiary Alkyl Halides. Synlett 2016, 27, 754-758.

(74) Bagal, S. K.; Brown, A. D.; Cox, P. J.; Omoto, K.; Owen, R. M.; Pryde, D. C.; Sidders, B.; Skerratt, S. E.; Stevens, E. B.; Storer, R. I.; et al. Ion Channels as Therapeutic Targets: A Drug Discovery Perspective. J. Med. Chem. 2013, 56, 
593-624.

(75) Guolin Wu†, Tingting Lv†, Wenhui Mo, Xiping Yang, Yu Gao, H. C. One-Pot Synthesis of Tricyclo-1,4-Benzoxazines Via Visible-Light Photoredox Catalysis In Continuous Flow. Tetrahedron Lett. 2017, 58, 1395-1398.

(76) Waisser, K.; Petrlíková, E.; Per̂ina, M.; Kliměová, V.; Kuně, J.; Palát, K.; Kaustová, J.; Dahse, H. M.; Möllmann, U. A Note to the Biological Activity of Benzoxazine Derivatives Containing the Thioxo Group. Eur. J. Med. Chem. 2010, 45, 2719-2725.

(77) Seo, H.; Katcher, M. H.; Jamison, T. F. Photoredox Activation of Carbon Dioxide for Amino Acid Synthesis in Continuous Flow. Nat. Chem. 2017, 9, 453456.

(78) Hone, C. A.; Roberge, D. M.; Kappe, C. O. The Use of Molecular Oxygen in Pharmaceutical Manufacturing: Is Flow the Way to Go? ChemSusChem 2017, $10,32-41$.

(79) Amara, Z.; Bellamy, J. F. B.; Horvath, R.; Miller, S. J.; Beeby, A.; Burgard, A.; Rossen, K.; Poliakoff, M.; George, M. W. Applying Green Chemistry to the Photochemical Route to Artemisinin. Nat. Chem. 2015, 7, 489-495.

(80) John E. Cochran and Nathan Waal. Photochemical Rearrangement of Chiral Oxaziridines in Continuous Flow: Application Toward the Scale-Up of a Chiral Bicyclic Lactam. Org. Process Res. Dev. 2016, 20, 1533-1539.

(81) Neitzel, M. L.; Aubele, D. L.; Marugg, J. L.; Jagodzinski, J. J.; Konradi, A. W.; Pleiss, M. A.; Szoke, B.; Zmolek, W.; Goldbach, E.; Quinn, K. P.; et al. Amino- 
Caprolactam $\gamma$-Secretase Inhibitors Showing Potential for the Treatment of Alzheimer's Disease. Bioorganic Med. Chem. Lett. 2011, 21, 3715-3720.

(82) Baumann, M.; Baxendale, I. R. Continuous Photochemistry: The Flow Synthesis of Ibuprofen via a Photo-Favorskii Rearrangement. React. Chem. Eng. 2016, 1, 147-150.

(83) Gérardy, R.; Winter, M.; Horn, C. R.; Vizza, A.; Van Hecke, K.; Monbaliu, J.-C. M. Continuous-Flow Preparation of $\gamma$-Butyrolactone Scaffolds from Renewable Fumaric and Itaconic Acids under Photosensitized Conditions. Org. Process Res. Dev. 2017, 21, 2012-2017.

(84) Nallasivam, J. L.; Fernandes, R. A. A Protecting-Group-Free Synthesis of (+)Nephrosteranic, (+)-Protolichesterinic, (+)-Nephrosterinic, (+)-Phaseolinic, (+)Rocellaric Acids and (+)-Methylenolactocin. Org. Biomol. Chem. 2017, 15, 708716.

(85) Gérardy, R.; Winter, M.; Vizza, A.; Monbaliu, J.-C. M. Assessing Inter- and Intramolecular Continuous-Flow Strategies towards Methylphenidate (Ritalin) Hydrochloride. React. Chem. Eng. 2017, 2, 149-158.

(86) Britton, J.; Raston, C. L. Multi-Step Continuous-Flow Synthesis. Chem. Soc. Rev. 2017, 46, 1250-1271.

(87) Greb, A.; Poh, J.-S.; Greed, S.; Battilocchio, C.; Pasau, P.; Blakemore, D. C.; Ley, S. V. A New Versatile Route to Unstable Diazo Compounds via Oxadiazolines and Use In Aryl-Alkyl Cross-Coupling Reactions. Angew. Chemie Int. Ed. 2017, 16602-16605. 
(88) Bottecchia, C.; Rubens, M.; Gunnoo, S. B.; Hessel, V.; Madder, A.; Noël, T. Visible-Light-Mediated Selective Arylation of Cysteine in Batch and Flow. Angew. Chemie - Int. Ed. 2017, 56, 12702-12707.

(89) Abdiaj, I.; Alcázar, J. Bioorganic \& Medicinal Chemistry Improving the Throughput of Batch Photochemical Reactions Using Flow: Dual Photoredox and Nickel Catalysis in Flow for C ( Sp 2 ) A C ( Sp 3 ) Cross- Coupling. Bioorg. Med. Chem. 2017, 25, 6190-6196.

(90) Qrareya, H.; Dondi, D.; Ravelli, D.; Fagnoni, M. Decatungstate-Photocatalyzed Si-H/C-H Activation in Silyl Hydrides: Hydrosilylation of Electron-Poor Alkenes. ChemCatChem 2015, 7, 3350-3357.

(91) Shen, B.; Jamison, T. F. Continuous Flow Photochemistry for the Rapid and Selective Synthesis of 2' -Deoxy and 2', $3^{\prime}$-Dideoxynucleosides. Aust. J. Chem. 2013, 66, 157-164.

(92) Colombeau, L.; Teste, K.; Hadj-Bouazza, A.; Chaleix, V.; Zerrouki, R.; Kraemer, M.; Catherine, O. Sainte. Synthesis and Biological Activity of Chloroethyl Pyrimidine Nucleosides. Nucleosides, Nucleotides and Nucleic Acids 2008, 27, 110-120.

(93) Kanishchev, O. S.; Gudz, G. P.; Shermolovich, Y. G.; Nesterova, N. V.; Zagorodnya, S. D.; Golovan, A. V. Synthesis and Biological Activity of the Nucleoside Analogs Based on Polyfluoroalkyl-Substituted 1,2,3-Triazoles. Nucleosides, Nucleotides and Nucleic Acids 2011, 30, 768-783.

(94) Fuse, S.; Otake, Y.; Nakamura, H. Integrated Micro-Flow Synthesis Based on Photochemical Wolff Rearrangement. European J. Org. Chem. 2017, 6466-6473. 
(95) Pinho, V. D.; Gutmann, B.; Kappe, C. O. Continuous Flow Synthesis of $\beta$-Amino Acids from $\alpha$-Amino Acids via Arndt-Eistert Homologation. RSC Adv. 2014, 4, 37419-37422.

(96) Yueh, H.; Voevodin, A.; Beeler, A. B. Development of a Photolabile Amine Protecting Group Suitable for Multistep Flow Synthesis. J. Flow Chem. 2015, 5, 155-159.

(97) Stringham, R. W.; Teager, D. S. Streamlined Process for the Conversion of Artemisinin to Artemether. Org. Process Res. Dev. 2012, 16, 764-768.

(98) Triemer, S.; Gilmore, K.; Vu, G. T.; Seeberger, P. H.; Seidel-Morgenstern, A. Literally Green Chemical Synthesis of Artemisinin from Plant Extracts. Angew. Chemie Int. Ed. 2018, 57, 5525-5528.

(99) Vukelić, S.; Koksch, B.; Seeberger, P. H.; Gilmore, K. A Sustainable, SemiContinuous Flow Synthesis of Hydantoins. Chem. - A Eur. J. 2016, 22, 1345113454.

(100) Parisien-Collette, S.; Collins, S. K. Exploiting Photochemical Processes in MultiStep Continuous Flow: Derivatization of the Natural Product Clausine C. ChemPhotoChem 2018, DOI: 10.1002/cptc.201800096. 